\title{
Approximate Conditional Least Squares Estimation of a Nonlinear State-Space Model via Unscented Kalman
}

\author{
Filter \\ Kwang Woo AhN \\ Division of Biostatistics \\ Medical College of Wisconsin, Milwaukee, WI 53226 \\ email: kwooahn@mcw.edu \\ Kung-Sik CHAN \\ Department of Statistics and Actuarial Science \\ The University of Iowa, Iowa City, Iowa 52242 \\ email: kung-sik-chan@uiowa.edu
}

July 22, 2011

\begin{abstract}
We consider the problem of estimating a nonlinear state-space model whose state process is driven by an ordinary differential equation (ODE) or a stochastic differential equation (SDE), with discrete-time data. We propose a new estimation method by minimizing the conditional least squares (CLS) with the conditional mean function computed approximately via unscented Kalman filter (UKF). We derive conditions
\end{abstract}


under which the UKF-CLS estimator preserves the limiting properties of the exact CLS estimator, namely, consistency and asymptotic normality. We demonstrate the efficacy of the proposed method by simulation and a real application.

KEYWORDS: Nonlinear time series; State-space model; Unscented Kalman filter; SIR model.

\section{INTRODUCTION}

The Kalman filter was proposed by Kalman (1960) who demonstrated the usefulness of the Kalman filter for drawing inference for a wide class of problems using the linear state-space model. However, many scientific studies require nonlinear state-space models with which the linear Kalman filter is inapplicable. For instance, the Susceptible-Infected-Recovered (SIR) model, which is the nonlinear ordinary differential equation, has been widely used in epidemiology, see Diekmann and Heesterbeek (2000).

Many statistical methods for estimating a nonlinear state-space model have been proposed in the literature. Durbin and Koopman (2000) proposed an iterative scheme for deriving an approximate linear-model whose likelihood approximately matches the mode of the log-likelihood function of the underlying nonlinear model. Using the linear approximate model, they proposed importance sampling schemes from classical and Bayesian perspectives. However, as Durbin and Koopman (2001) pointed out, the estimated modes need not be the same as the true modes due to linearization. Also their method suffers from the need for calculating the Jacobian matrix, which may not be available. Other approaches, for example, particle filter, bootstrap filter, and sequential Monte Carlo, have been proposed in engineering and statistics, see Kitagawa (1996), Liu and Chen (1998), and Pitt and Shephard (1999). See Doucet, deFreitas and Gordon (2001) for a comprehensive survey. Starting with random samples called particles from an initial distribution, these methods update the particles according to the conditional or the posterior distribution of the true states based on importance sampling, which is often computationally expensive as Durbin and Koopman (2001). On the other hand, the extended Kalman filter (EKF) has become a standard 
technique in the engineering literature. The EKF approximates a nonlinear model by its first order Taylor expansion, which is similar to the delta method. However, the EKF has some serious defects. First, the approximation error becomes non-negligible with strongly nonlinear models. Another drawback is that similar to the method of Durbin and Koopman (2000), the EKF requires calculating the Jacobian, which may not be available or be very complex.

Besides nonlinear least squares, several methods have been proposed for specifically estimating a nonlinear state-space model driven by an ordinary differential equation (ODE), with observations being some function of the state vector corrupted by independent and identically distributed (iid) errors. Ramsay, Hooker, Campbell and Cao (2007) proposed the generalized profile estimation method. However, the method is computationally expensive as it requires profiling out the generally high-dimensional coefficient in the functional expansion. Liang and Wu (2008) proposed the two-step approach that consists of (i) estimating the derivatives of the state process from data at the sampling epochs, via some nonparametric smoothing methods, and then (ii) estimating the model parameters by minimizing the sum of squared deviations between the left and right side of the defining ODE, with the derivatives replaced by their nonparametric estimates from (i), but it is applicable only when the full state vector is observed with measurement errors over discrete time.

Instead of relying on linearization techniques employed by the EKF, the unscented Kalman filter (UKF) was proposed by Julier and Uhlmann (1997) for extending Kalman filter to nonlinear models using a deterministic sampling scheme consisting of the so-called "sigma points." In each step, the UKF generates a set of sigma points and updates the prediction formulas based on these sigma points. So the UKF bears resemblance to the particle filter. However, the sigma points are deterministic samples and the number of the sigma points is much less than the number of particles. Consequently, the UKF is computationally more efficient than the particle filter. Also, empirical results suggest that the UKF appears to be superior to EKF and works properly and satisfactorily from a Bayesian perspective, see Julier and Uhlmann (2004) and Wu and Smyth (2007). Yet the use of UKF in estimating a 
nonlinear state-space model from a frequentist perspective is largely unexplored.

The method of conditional least squares (CLS) provides a general approach for estimating a state-space model and it enjoys consistency and asymptotic normality, under some mild regularity conditions, see Klimko and Nelson (1978). However, its applicability is limited by the tractability of the conditional mean function of the future process given the data, which is generally intractable for nonlinear state-space models. The UKF provides a numerical scheme for computing the predictors with which we can calculate an approximation to the conditional least squares (CLS) objective function. We propose to estimate a model by minimizing the latter objective function; we shall refer to this method as the UKF-CLS method. The main purpose of our paper is to study conditions under which the UKFCLS estimator preserves the consistency and the asymptotic distribution of the exact CLS estimator.

In Section 2, we briefly review the limiting properties of the CLS method and show that the approximate CLS method preserves the asymptotic properties of the CLS method if the approximation scheme of the conditional mean function satisfies a certain convergence rate. We detail the UT and UKF in Section 3. In order for the proposed UKF-CLS approach to work, it is pivotal to study the convergence rate of the UKF in terms of estimating the predictive mean, which we address in Sections 4 and 5. We are particularly interested in estimating a state-space model whose state process is driven by some ordinary differential equation (ODE) or stochastic differential equation (SDE), in which case, the model can be discretized with arbitrarily fine time step. In Section 5, we study that convergence rate of the UKF as the discretization time step converges to 0 , and show that, under some regularity conditions, the error rate of the UKF estimation scheme is of sufficiently high order that the UKF-CLS estimator enjoys the same asymptotic distribution of the exact CLS estimator. The efficacy of the proposed method is then illustrated by simulations in Section 7 and a real application in Section 8. We briefly conclude in Section 9. 


\section{APPROXIMATE CLS}

Let the conditional expectation of $\mathbf{y}_{t}$ given $\mathbf{y}_{1}, \ldots, \mathbf{y}_{t-1}$ be denoted by $\mathrm{E}_{\boldsymbol{\theta}}\left(\mathbf{y}_{t} \mid \mathbf{y}_{1}, \ldots, \mathbf{y}_{t-1}\right)=$ $\mathrm{E}_{\boldsymbol{\theta}}\left(\mathbf{y}_{t \mid t-1}\right)$; the conditional mean is the best 1-step ahead predictor in the sense of minimizing the mean squared prediction error. Then, the CLS method estimates the $a \times 1$ unknown true parameter value $\boldsymbol{\theta}_{0}$ by the argument, denoted by $\hat{\boldsymbol{\theta}}$, that minimizes the conditional sum of squares

$$
Q_{n}(\boldsymbol{\theta})=\sum_{t=1}^{n}\left(\mathbf{y}_{t}-\mathrm{E}_{\boldsymbol{\theta}}\left(\mathbf{y}_{t \mid t-1}\right)\right)^{2} .
$$

Klimko and Nelson (1978, Theorem 2.2) have established some general large-sample properties for the CLS estimator, which we now briefly describe. Let $|\cdot|$ be the Euclidean norm. Assume that the conditional expectation $\mathrm{E}_{\boldsymbol{\theta}}\left(\mathbf{y}_{t \mid t-1}\right)$ is twice continuously differentiable in $\boldsymbol{\theta}$. Taylor expansion implies that for $\delta>0$ and $\left|\boldsymbol{\theta}-\boldsymbol{\theta}_{0}\right|<\delta$, there exists $\boldsymbol{\theta}^{*}$ with $\left|\boldsymbol{\theta}_{0}-\boldsymbol{\theta}^{*}\right|<\delta$ such that

$$
\begin{aligned}
Q_{n}(\boldsymbol{\theta})= & Q_{n}\left(\boldsymbol{\theta}_{0}\right)+\left(\boldsymbol{\theta}-\boldsymbol{\theta}_{0}\right)^{T} \frac{\partial Q_{n}\left(\boldsymbol{\theta}_{0}\right)}{\partial \boldsymbol{\theta}}+\frac{1}{2}\left(\boldsymbol{\theta}-\boldsymbol{\theta}_{0}\right)^{T} \mathbf{V}_{n}\left(\boldsymbol{\theta}-\boldsymbol{\theta}_{0}\right) \\
& +\frac{1}{2}\left(\boldsymbol{\theta}-\boldsymbol{\theta}_{0}\right)^{T} \mathbf{U}_{n}\left(\boldsymbol{\theta}^{*}\right)\left(\boldsymbol{\theta}-\boldsymbol{\theta}_{0}\right)
\end{aligned}
$$

where

$$
\mathbf{V}_{n}=\left(\frac{\partial^{2} Q_{n}\left(\boldsymbol{\theta}_{0}\right)}{\partial \theta_{i} \partial \theta_{j}}\right), \mathbf{U}_{n}\left(\boldsymbol{\theta}^{*}\right)=\left(\frac{\partial^{2} Q_{n}\left(\boldsymbol{\theta}^{*}\right)}{\partial \boldsymbol{\theta}^{2}}-\mathbf{V}_{n}\right)
$$

Under the following four assumptions:

A1 $\frac{1}{2 n} \mathbf{V}_{n} \rightarrow \mathbf{V}$ a.e., a fixed positive definite matrix,

A2 $\lim _{n \rightarrow \infty} \sup _{\delta \rightarrow 0}\left(\frac{\left|U_{n}\left(\boldsymbol{\theta}^{*}\right)_{i j}\right|}{n \delta}\right)<\infty, i \leq a, j \leq a$,

A3 $\frac{1}{n} \frac{\partial Q_{n}\left(\boldsymbol{\theta}_{0}\right)}{\partial \theta_{i}} \rightarrow 0$ a.e., $i \leq a$,

$\mathbf{A} 4 \frac{1}{2 \sqrt{n}} \frac{\partial Q_{n}\left(\boldsymbol{\theta}_{0}\right)}{\partial \theta_{i}} \rightsquigarrow \mathrm{N}(\mathbf{0}, \mathbf{W})$,

Klimko and Nelson (1978) derived the consistency and the large sample distribution of $\hat{\boldsymbol{\theta}}$ :

$$
\sqrt{n}\left(\hat{\boldsymbol{\theta}}-\boldsymbol{\theta}_{0}\right) \rightsquigarrow \mathrm{N}\left(\mathbf{0}, \mathbf{V}^{-1} \mathbf{W} \mathbf{V}^{-1}\right)
$$

Specifically, Klimko and Nelson (1978) showed the existence of a local minimizer of the objective function in the interior of a shrinking neighborhood of the true parameter, with 
probability approaching 1 as sample size $n \rightarrow \infty$, and the local minimizer constitutes the CLS estimator which is then shown to be asymptotically normal.

The CLS method is, however, not always applicable for estimating a nonlinear state-space model because the 1-step ahead predictor is generally intractable. This problem may be overcome if the 1-step ahead predictor can be well approximated by some numerical scheme. We show below that the approximate CLS estimator enjoys the same large-sample properties of the (exact) CLS estimator, if the approximation error is of the order $o_{p}\left(n^{-1}\right)$. Moreover, we shall demonstrate in latter sections that the UKF provides such an approximation scheme. Let $\hat{\mathrm{E}}_{\boldsymbol{\theta}}\left(\mathbf{y}_{t \mid t-1}\right)$ be an approximation of $\mathrm{E}_{\boldsymbol{\theta}}\left(\mathbf{y}_{t \mid t-1}\right)$. Then, we have the following theorem:

Theorem 1. Assume

(i) there exist a real number $r \geq 1$ and a neighborhood of the true parameter value, denoted by $W$, such that $E_{\boldsymbol{\theta}}\left(\mathbf{y}_{t \mid t-1}\right)-\hat{E}_{\boldsymbol{\theta}}\left(\mathbf{y}_{t \mid t-1}\right)=o_{p}\left(n^{-r}\right)$ uniformly for all $t$ and for all $\boldsymbol{\theta} \in W$;

(ii) the conditional variance of $\mathbf{y}_{t}$ given $\mathbf{y}_{1}, \ldots, \mathbf{y}_{t-1}$ under $\boldsymbol{\theta}_{0}$, denoted by $\sigma_{t \mid t-1}^{2}$, is uniformly bounded for all $t$.

Then,

$$
\frac{1}{n} \sum_{t=1}^{n}\left(\mathbf{y}_{t}-\hat{E}_{\boldsymbol{\theta}}\left(\mathbf{y}_{t \mid t-1}\right)\right)^{2}=\frac{1}{n} \sum_{t=1}^{n}\left(\mathbf{y}_{t}-E_{\boldsymbol{\theta}}\left(\mathbf{y}_{t \mid t-1}\right)\right)^{2}+o_{p}\left(n^{-r}\right)
$$

and the approximate CLS estimator enjoys the same consistency and asymptotic normality result of the exact CLS estimators of Klimko and Nelson (1978).

Proof. Consider the normalized approximate conditional sum of squared prediction error function:

$$
\frac{\hat{Q}_{n}(\boldsymbol{\theta})}{n}=\frac{1}{n} \sum_{t=1}^{n}\left(\mathbf{y}_{t}-\hat{\mathrm{E}}_{\boldsymbol{\theta}}\left(\mathbf{y}_{t \mid t-1}\right)\right)^{2}
$$


Consider the difference

$$
\begin{aligned}
& \frac{1}{n} \sum_{t=1}^{n}\left(\mathbf{y}_{t}-\hat{\mathrm{E}}_{\boldsymbol{\theta}}\left(\mathbf{y}_{t \mid t-1}\right)\right)^{2}-\frac{1}{n} \sum_{t=1}^{n}\left(\mathbf{y}_{t}-\mathrm{E}_{\boldsymbol{\theta}}\left(\mathbf{y}_{t \mid t-1}\right)\right)^{2} \\
= & \frac{1}{n} \sum_{t=1}^{n}\left\{\mathrm{E}_{\boldsymbol{\theta}}\left(\mathbf{y}_{t \mid t-1}\right)-\hat{\mathrm{E}}_{\boldsymbol{\theta}}\left(\mathbf{y}_{t \mid t-1}\right)\right\}\left\{2 \mathbf{y}_{t}-\hat{\mathrm{E}}_{\boldsymbol{\theta}}\left(\mathbf{y}_{t \mid t-1}\right)-\mathrm{E}_{\boldsymbol{\theta}}\left(\mathbf{y}_{t \mid t-1}\right)\right\} \\
= & \frac{2}{n} \sum_{t=1}^{n}\left\{\mathrm{E}_{\boldsymbol{\theta}}\left(\mathbf{y}_{t \mid t-1}\right)-\hat{\mathrm{E}}_{\boldsymbol{\theta}}\left(\mathbf{y}_{t \mid t-1}\right)\right\}\left\{\mathbf{y}_{t}-\mathrm{E}_{\boldsymbol{\theta}}\left(\mathbf{y}_{t \mid t-1}\right)\right\}+\frac{1}{n} \sum_{t=1}^{n}\left\{\mathrm{E}_{\boldsymbol{\theta}}\left(\mathbf{y}_{t \mid t-1}\right)-\hat{\mathrm{E}}_{\boldsymbol{\theta}}\left(\mathbf{y}_{t \mid t-1}\right)\right\}^{2} .
\end{aligned}
$$

Below, the last two summands are written as $A$ and $B$, respectively. It follows from (ii) that $\sum_{t=1}^{n}\left\{\mathbf{y}_{t}-\mathrm{E}_{\boldsymbol{\theta}_{0}}\left(\mathbf{y}_{t \mid t-1}\right)\right\}^{2} / n=O_{p}(1)$. An application of Cauchy-Schwartz inequality then implies that $A=o_{p}\left(n^{-r}\right)$, uniformly for $\boldsymbol{\theta} \in W$, owing to (i). Similarly, $B=o_{p}\left(n^{-2 r}\right)$, uniformly for $\boldsymbol{\theta} \in W$. Therefore, uniformly for $\boldsymbol{\theta} \in W$,

$$
\frac{1}{n} \sum_{t=1}^{n}\left(\mathbf{y}_{t}-\hat{\mathrm{E}}_{\boldsymbol{\theta}}\left(\mathbf{y}_{t \mid t-1}\right)\right)^{2}=\frac{1}{n} \sum_{t=1}^{n}\left(\mathbf{y}_{t}-\mathrm{E}_{\boldsymbol{\theta}}\left(\mathbf{y}_{t \mid t-1}\right)\right)^{2}+o_{p}\left(n^{-r}\right) .
$$

Since $r \geq 1$, the approximate CLS estimator enjoys the same consistency and asymptotic normality result of the exact CLS estimators thanks to Theorem 2.1, Corollary 2.1, and Theorem 2.2 of Klimko and Nelson (1978) and Theorem 5.23 of van der Vaart (1998).

We will show that the case of $r \geq 1$ can be achieved via the UKF, in Sections 5 and 6 .

\section{UNSCENTED KALMAN FILTER}

\subsection{Unscented Transformation}

For a linear function $f(\mathbf{x})$, the identity $\mathrm{E}[f(\mathbf{x})]=f(\mathrm{E}(\mathbf{x}))$ holds. This property makes it straightforward to calculate the conditional means and covariance matrices for a linear Gaussian state-space model. However, if $f(\mathbf{x})$ is nonlinear, the preceding identity is generally invalid. The unscented transformation (UT) is an approximate scheme for computing the mean and covariance matrix of $\mathbf{y}=f(\mathbf{x})$. Let $\mathbf{x}$ be a $c \times 1$ random vector with mean $\mathrm{E}(\mathbf{x})$ and covariance matrix $\mathbf{P}_{\mathbf{x}}$. Let $\lambda>-c$ be a constant. The UT approximates $E\{f(\mathbf{x})\}$ via a particular sample of $\mathbf{x}$, of size $2 c$, that are referred to as sigma points and defined by the 
following equations:

$$
\begin{aligned}
\hat{\mathbf{x}}^{(0)} & =\mathrm{E}(\mathbf{x}), \\
\hat{\mathbf{x}}^{(i)} & =\mathrm{E}(\mathbf{x})+\left(\sqrt{(c+\lambda) \mathbf{P}_{\mathbf{x}}}\right)_{i}^{T}, \\
\hat{\mathbf{x}}^{(i+c)} & =\mathrm{E}(\mathbf{x})-\left(\sqrt{(c+\lambda) \mathbf{P}_{\mathbf{x}}}\right)_{i}^{T},
\end{aligned}
$$

where $i=1, \ldots, c$ and $\sqrt{(c+\lambda) \mathbf{P}_{\mathbf{x}}}$ is the matrix square root of $(c+\lambda) \mathbf{P}_{\mathbf{x}}$ such that

$$
\left(\sqrt{(c+\lambda) \mathbf{P}_{\mathbf{x}}}\right)^{T}\left(\sqrt{(c+\lambda) \mathbf{P}_{\mathbf{x}}}\right)=(c+\lambda) \mathbf{P}_{\mathbf{x}}
$$

and $\left(\sqrt{(c+\lambda) \mathbf{P}_{\mathbf{x}}}\right)_{i}$ is the $i$ th row of $\sqrt{(c+\lambda) \mathbf{P}_{\mathbf{x}}}$. The constant $\lambda$ is a tuning parameter that controls the distance between the sigma points and $\mathrm{E}(\mathbf{x})$. The sigma points tend to be closer to $\mathrm{E}(\mathbf{x})$, for $-c<\lambda \leq 0$, but, otherwise, they tend to be further away from $\mathrm{E}(\mathbf{x})$. How to choose an optimal $\lambda$ is a difficult problem; see Ahn and Chan (2011). Here, for simplicity, we set $\lambda$ to 0 in all numerical work reported below. The approximate mean $\hat{\mathbf{y}}$, covariance matrix $\hat{\mathbf{P}}_{\mathbf{y}}$, and covariance matrix $\hat{\mathbf{P}}_{\mathbf{x y}}$ between $\mathbf{x}$ and $\mathbf{y}$ from the UT are defined as follows:

$$
\begin{aligned}
\hat{\mathbf{y}} & =\sum_{i=0}^{2 c} W^{(i)} \hat{\mathbf{y}}^{(i)}, \quad \hat{\mathbf{P}}_{\mathbf{y}}=\sum_{i=0}^{2 c} W^{(i)}\left(\hat{\mathbf{y}}^{(i)}-\hat{\mathbf{y}}\right)\left(\hat{\mathbf{y}}^{(i)}-\hat{\mathbf{y}}\right)^{T}, \\
\hat{\mathbf{P}}_{\mathbf{x y}} & =\sum_{i=0}^{2 c} W^{(i)}\left(\hat{\mathbf{x}}^{(i)}-\mathrm{E}(\mathbf{x})\right)\left(\hat{\mathbf{y}}^{(i)}-\hat{\mathbf{y}}\right)^{T},
\end{aligned}
$$

where $\hat{\mathbf{y}}^{(i)}=f\left(\hat{\mathbf{x}}^{(i)}\right), i=0, \ldots, 2 c$ and

$$
W^{(0)}=\frac{\lambda}{c+\lambda}, W^{(j)}=\frac{1}{2(c+\lambda)}, j=1, \ldots, 2 c .
$$

Hence, the UT is a weighted average scheme based on the sigma points, see Simon (2006). On the other hand, the linear approximation scheme used by the EKF approximates the mean and covariance matrices via the first order Taylor expansion, resulting in the following formulas:

$$
\hat{\mathbf{y}}_{L}=f\{\mathrm{E}(\mathbf{x})\}, \hat{\mathbf{P}}_{\mathbf{y}, L}=\mathbf{Z} \mathbf{P}_{\mathbf{x}} \mathbf{Z}^{T}, \hat{\mathbf{P}}_{\mathbf{x y}, L}=\mathbf{P}_{\mathbf{x}} \mathbf{Z}^{T}
$$

where $\mathbf{Z}$ is the Jacobian matrix of $f$ evaluated at $\mathrm{E}(\mathbf{x})$. 


\subsection{Unscented Kalman Filter}

The UKF is an iterative scheme for computing the one-step ahead predictors (not necessarily the conditional means) and the covariance matrices of the prediction errors for a nonlinear state-space model. Consider the following state-space model:

$$
\begin{aligned}
\mathbf{y}_{t_{k}} & =u_{t_{k}}\left(\mathbf{x}_{t_{k}}\right)+\varepsilon_{t_{k}}, \quad \varepsilon_{t_{k}} \sim\left(0, \mathbf{H}_{t_{k}}\right), \\
\mathbf{x}_{t_{k+1}} & =v_{t_{k}}\left(\mathbf{x}_{t_{k}}\right)+\boldsymbol{\eta}_{t_{k}}, \quad \boldsymbol{\eta}_{t_{k}} \sim\left(0, \mathbf{Q}_{t_{k}}\right), \\
\mathbf{x}_{0} & \sim\left(\mathrm{E}\left(\mathbf{x}_{0}\right), \mathbf{Q}_{0}\right),
\end{aligned}
$$

where $u_{t_{k}} \equiv 1$ when $\mathbf{y}_{t_{k}}$ is missing and $t_{k+1}-t_{k} \equiv h$, for some constant $h>0$. Here, $\mathbf{y}_{t_{k}}$ is a $q \times 1$ vector of observations and $\mathbf{x}_{t_{k}}$ is the corresponding $c \times 1$ state vector. The error terms $\boldsymbol{\varepsilon}_{t_{k}}$ and $\boldsymbol{\eta}_{t_{k}}$ are assumed to be serially independent and independent of each other at all time points. Note that we do not assume any specific distributions for the error terms and the initial state vector. This model formulation can also be useful for modeling discretetime data sampled from a continuous-time model, e.g., a differential equation model or a stochastic differential equation model. For continuous-time models, the above state-space model may be obtained by a discrete-time approximation of the underlying continuous-time process, in which case we may update the true states multiple times, say $s$ times, between two consecutive observations. Below, $t_{0}$ and $T$ denote the initial and final time points, respectively.

The UKF algorithm attempts to compute the conditional mean of $\mathbf{y}_{t_{k}}$ given observations up to and including time $t_{k-1}$, denoted by $\hat{\mathbf{y}}_{t_{k} \mid t_{k-1}}$, and $\hat{\mathbf{P}}_{\mathbf{y}, t_{k} \mid t_{k-1}}$, the corresponding covariance matrix of the prediction error; like Kalman filter, the algorithm also needs to compute the counterparts for $\mathbf{x}_{t_{k}}$, e.g. the conditional mean $\hat{\mathbf{x}}_{t_{k} \mid t_{k-1}}$, etc.

We now describe the UKF updating scheme (Simon 2006):

1. The UKF is initialized as follows:

$$
\hat{\mathbf{x}}_{0 \mid 0}=\mathrm{E}\left(\mathbf{x}_{0}\right), \hat{\mathbf{P}}_{\mathbf{x}, 0 \mid 0}=\mathrm{E}\left[\left(\mathbf{x}_{0}-\hat{\mathbf{x}}_{0 \mid 0}\right)\left(\mathbf{x}_{0}-\hat{\mathbf{x}}_{0 \mid 0}\right)^{T}\right]
$$

2. Update the state vector. 
(a) Let the estimators of the mean and the covariance matrix of $\mathbf{x}_{t_{k-1}}$ given $\left\{\mathbf{y}_{t_{i}}, i<\right.$ $k\}$ be $\hat{\mathbf{x}}_{t_{k-1} \mid t_{k-1}}$ and $\hat{\mathbf{P}}_{\mathbf{x}, t_{k-1} \mid t_{k-1}}$, respectively. Let $\lambda>-c$ be a constant. For $i=1, \ldots, c$, define the sigma points:

$$
\begin{aligned}
& \hat{\mathbf{x}}_{t_{k-1} \mid t_{k-1}}^{(0)}=\hat{\mathbf{x}}_{t_{k-1} \mid t_{k-1}}, \\
& \hat{\mathbf{x}}_{t_{k-1} \mid t_{k-1}}^{(i)}=\hat{\mathbf{x}}_{t_{k-1} \mid t_{k-1}}+\left(\sqrt{(c+\lambda) \hat{\mathbf{P}}_{\mathbf{x}, t_{k-1} \mid t_{k-1}}}\right)_{i}^{T}, \\
& \hat{\mathbf{x}}_{t_{k-1} \mid t_{k-1}}^{(i+c)}=\hat{\mathbf{x}}_{t_{k-1} \mid t_{k-1}}-\left(\sqrt{(c+\lambda) \hat{\mathbf{P}}_{\mathbf{x}, t_{k-1} \mid t_{k-1}}}\right)_{i}^{T} .
\end{aligned}
$$

(b) The UKF updates the conditional mean of $\mathbf{x}_{t_{k}}$ and the corresponding predictive error covariance matrix approximately by

$$
\begin{aligned}
\hat{\mathbf{x}}_{t_{k} \mid t_{k-1}} & =\sum_{i=0}^{2 c} W^{(i)} \hat{\mathbf{x}}_{t_{k} \mid t_{k-1}}^{(i)}, \\
\hat{\mathbf{P}}_{\mathbf{x}, t_{k} \mid t_{k-1}} & =\sum_{i=0}^{2 c} W^{(i)}\left(\hat{\mathbf{x}}_{t_{k} \mid t_{k-1}}^{(i)}-\hat{\mathbf{x}}_{t_{k} \mid t_{k-1}}\right)\left(\hat{\mathbf{x}}_{t_{k} \mid t_{k-1}}^{(i)}-\hat{\mathbf{x}}_{t_{k} \mid t_{k-1}}\right)^{T}+\mathbf{Q}_{t_{k-1}},
\end{aligned}
$$

where $\hat{\mathbf{x}}_{t_{k} \mid t_{k-1}}^{(i)}=v_{t_{k-1}}\left(\hat{\mathbf{x}}_{t_{k-1} \mid t_{k-1}}^{(i)}\right)$ for $i=0,1, \ldots, 2 c$.

3. Implement the measurement update equations.

(a) Based on the estimators of the conditional mean and the covariance matrix, for $i=1, \ldots, c$ we choose the new sigma points as follows:

$$
\begin{aligned}
& \hat{\mathbf{x}}_{t_{k} \mid t_{k-1}}^{(0)+}=\hat{\mathbf{x}}_{t_{k} \mid t_{k-1}}, \\
& \hat{\mathbf{x}}_{t_{k} \mid t_{k-1}}^{(i)+}=\hat{\mathbf{x}}_{t_{k} \mid t_{k-1}}+\left(\sqrt{(c+\lambda) \hat{\mathbf{P}}_{\mathbf{x}, t_{k} \mid t_{k-1}}}\right)_{i}^{T}, \\
& \hat{\mathbf{x}}_{t_{k} \mid t_{k-1}}^{(i+c)+}=\hat{\mathbf{x}}_{t_{k} \mid t_{k-1}}-\left(\sqrt{(c+\lambda) \hat{\mathbf{P}}_{\mathbf{x}, t_{k} \mid t_{k-1}}}\right)_{i}^{T} .
\end{aligned}
$$

(b) The UKF then updates $\mathrm{E}\left(\mathbf{y}_{t_{k} \mid t_{k-1}}\right), \operatorname{Cov}\left(\mathbf{y}_{t_{k} \mid t_{k-1}}\right)$, and $\operatorname{Cov}\left(\mathbf{y}_{t_{k} \mid t_{k-1}}, \mathbf{y}_{t_{k} \mid t_{k-1}}\right)$ approximately by

$$
\begin{aligned}
\hat{\mathbf{y}}_{t_{k} \mid t_{k-1}} & =\sum_{i=0}^{2 c} W^{(i)} \hat{\mathbf{y}}_{t_{k} \mid t_{k-1}}^{(i)}, \\
\hat{\mathbf{P}}_{\mathbf{y}, t_{k} \mid t_{k-1}} & =\sum_{i=0}^{2 c} W^{(i)}\left(\hat{\mathbf{y}}_{t_{k} \mid t_{k-1}}^{(i)}-\hat{\mathbf{y}}_{t_{k} \mid t_{k-1}}\right)\left(\hat{\mathbf{y}}_{t_{k} \mid t_{k-1}}^{(i)}-\hat{\mathbf{y}}_{t_{k} \mid t_{k-1}}\right)^{T}+\mathbf{H}_{t_{k}} \\
\hat{\mathbf{P}}_{\mathbf{x y}, t_{k} \mid t_{k-1}} & =\sum_{i=0}^{2 c} W^{(i)}\left(\hat{\mathbf{x}}_{t_{k} \mid t_{k-1}}^{(i)}-\hat{\mathbf{x}}_{t_{k} \mid t_{k-1}}\right)\left(\hat{\mathbf{y}}_{t_{k} \mid t_{k-1}}^{(i)}-\hat{\mathbf{y}}_{t_{k} \mid t_{k-1}}\right)^{T}
\end{aligned}
$$


where $\hat{\mathbf{y}}_{t_{k} \mid t_{k-1}}^{(i)}=u_{t_{k}}\left(\hat{\mathbf{x}}_{t_{k} \mid t_{k-1}}^{(i)+}\right)$ for $i=0, \ldots, 2 c$.

(c) $\mathrm{E}\left(\mathbf{x}_{t_{k} \mid t_{k}}\right)$ and $\operatorname{Cov}\left(\mathbf{x}_{t_{k} \mid t_{k}}\right)$ are estimated by

$$
\begin{aligned}
\mathbf{K}_{t_{k} \mid t_{k-1}} & =\hat{\mathbf{P}}_{\mathbf{x y}, t_{k} \mid t_{k-1}} \hat{\mathbf{P}}_{\mathbf{y}, t_{k} \mid t_{k-1}}^{-1}, \\
\hat{\mathbf{x}}_{t_{k} \mid t_{k}} & =\hat{\mathbf{x}}_{t_{k} \mid t_{k-1}}+\mathbf{K}_{t_{k} \mid t_{k-1}}\left(\mathbf{y}_{t_{k}}-\hat{\mathbf{y}}_{t_{k} \mid t_{k-1}}\right) \\
\hat{\mathbf{P}}_{\mathbf{x}, t_{k} \mid t_{k}} & =\hat{\mathbf{P}}_{\mathbf{x}, t_{k} \mid t_{k-1}}-\mathbf{K}_{t_{k} \mid t_{k-1}} \hat{\mathbf{P}}_{\mathbf{y}, t_{k} \mid t_{k-1}} \mathbf{K}_{t_{k} \mid t_{k-1}}^{T}
\end{aligned}
$$

4. Repeat step 2 and 3 for $t_{k}=t_{0}, \ldots, T$.

The UKF has several attractive properties. First, it is computationally more efficient than simulation based methods. Also the UKF does not require calculating the Jacobian matrix and it provides more accurate approximation than the EKF, see Section 4 and 5 .

\section{CONVERGENCE RATE OF THE UT}

Before studying the asymptotics of the UKF, we review some convergence properties of the UT recently obtained by Ahn and Chan (2011). Let $\Omega$, a subset some Euclidean space, be the sample space of $\mathbf{x}$, and define $D_{k}$ as follows:

$$
D_{k}=\left\{f: \Omega \subset \mathbb{R}^{c} \rightarrow \mathbb{R}^{q} \mid f \text { is a polynomial of degree at most } k\right\}
$$

Then, Ahn and Chan (2011) proved the following two theorems on the UT estimators of the first two moments of $\mathbf{y}=f(\mathbf{x})$ :

Theorem 2. 1. If $f \in D_{2}$, then $\hat{\mathbf{y}}=E(\mathbf{y})$. In addition, if the distribution of $\mathbf{x}$ is symmetric about its mean and $f \in D_{3}$, then, $\hat{\mathbf{y}}=E(\mathbf{y})$.

2. If $f \in D_{1}$, i.e., $f$ is linear, then $\hat{\mathbf{P}}_{\mathbf{y}}=\mathbf{P}_{\mathbf{y}}$ and $\hat{\mathbf{P}}_{\mathbf{x y}}=\mathbf{P}_{\mathbf{x y}}$.

Theorem 3. Suppose $\mathbf{P}=h \mathbf{P}^{*}$ and $\mathbf{x}$ has a symmetric distribution about its mean. Assume

1. there exists a $h$-free constant $M>0$ such that $E\left|\hat{x}_{1}^{k_{1}} \cdots \hat{x}_{c}^{k_{c}}\right| \leq h^{i / 2} M^{i / 2}$, for any nonnegative integers $k_{j}$ where $\sum_{j=1}^{c} k_{j}=i$; 
2. for all $j, 1 \leq j \leq q$, and for all $0<h \leq 1$, there exists some $h$-free constant $K>0$,

$$
\left|\frac{\partial^{i} f_{j}(E(\mathbf{x}))}{\partial x_{1}^{k_{1}} \cdots \partial x_{c}^{k_{c}}}\right| \leq K^{i}
$$

for any non-negative integers $k_{j}, 1 \leq j \leq c$, where $i=\sum_{j=1}^{c} k_{j}$.

Then,

$$
\begin{aligned}
& E(\mathbf{y})-\hat{\mathbf{y}}=O\left(h^{2}\right), \mathbf{P}_{\mathbf{y}}-\hat{\mathbf{P}}_{\mathbf{y}}=O\left(h^{2}\right), \mathbf{P}_{\mathbf{x y}}-\hat{\mathbf{P}}_{\mathbf{x y}}=O\left(h^{2}\right), \\
& E(\mathbf{y})-\hat{\mathbf{y}}_{L}=O(h), \mathbf{P}_{\mathbf{y}}-\hat{\mathbf{P}}_{\mathbf{y}, L}=O\left(h^{2}\right), \mathbf{P}_{\mathbf{x y}}-\hat{\mathbf{P}}_{\mathbf{x y}, L}=O\left(h^{2}\right) .
\end{aligned}
$$

Note that Condition 1 of Theorem 3 is motivated by the fact that $\hat{x}_{1}^{k_{1}} \cdots \hat{x}_{c}^{k_{c}}=O_{p}\left(h^{i / 2}\right)$ because $\tilde{x}_{j}=O_{p}(\sqrt{h})$, under the condition $\mathbf{P}=h \mathbf{P}^{*}$. Condition 2 of Theorem 3 is satisfied if the function $f$ is a polynomial.

\section{CONVERGENCE RATE OF THE UKF}

In this section, we study the asymptotic properties of the UKF. Throughout this section, we assume the following state-space model that is identical to (3), except that now the coefficient matrices of the model depends on an unknown parameter $\boldsymbol{\theta}$ belonging to some parameter space $\Theta$ :

$$
\begin{aligned}
\mathbf{y}_{t_{k}} & =u_{t_{k}}\left(\mathbf{x}_{t_{k}}\right)+\varepsilon_{t_{k}}, \quad \varepsilon_{t_{k}} \sim\left(0, \mathbf{H}_{t_{k}}\right), \\
\mathbf{x}_{t_{k+1}} & =v_{t_{k}}\left(\mathbf{x}_{t_{k}}\right)+\boldsymbol{\eta}_{t_{k}}, \quad \boldsymbol{\eta}_{t_{k}} \sim\left(0, \mathbf{Q}_{t_{k}}\right), \\
\mathbf{x}_{0} & \sim\left(\mathrm{E}\left(\mathbf{x}_{0}\right), \mathbf{Q}_{0}\right) .
\end{aligned}
$$

The unknown parameter can be can be estimated by minimizing the sum of squared prediction error $\sum_{t_{k}=t_{0}}^{T}\left|\mathbf{y}_{t_{k}}-\hat{\mathbf{y}}_{t_{k} \mid t_{k-1}}(\boldsymbol{\theta})\right|^{2}$ where $\hat{\mathbf{y}}_{t_{k} \mid t_{k-1}}(\boldsymbol{\theta})$ is approximately computed by the UKF. Recall the 1-step ahead predictive distributions can be sequentially updated as follows (with distribution of the right side of a $\rightarrow$ sign updated from the distribution of its left side and additional data indicated by the subscripts):

$$
\begin{aligned}
& \mathbf{x}_{0} \equiv \mathbf{x}_{t_{0} \mid t_{0}} \rightarrow \mathbf{x}_{t_{1} \mid t_{0}} \rightarrow \mathbf{y}_{t_{1} \mid t_{0}} ;\left(\mathbf{x}_{t_{1} \mid t_{0}}, \mathbf{y}_{t_{1} \mid t_{0}}\right) \rightarrow \mathbf{x}_{t_{1} \mid t_{1}} \rightarrow \mathbf{x}_{t_{2} \mid t_{1}} \rightarrow \cdots \rightarrow \\
& \mathbf{x}_{t_{k} \mid t_{k}} \rightarrow \mathbf{x}_{t_{k+1} \mid t_{k}} \rightarrow \mathbf{y}_{t_{k+1} \mid t_{k}} ;\left(\mathbf{x}_{t_{k+1} \mid t_{k}}, \mathbf{y}_{t_{k+1} \mid t_{k}}\right) \rightarrow \mathbf{x}_{t_{k+1} \mid t_{k+1}} \rightarrow \mathbf{x}_{t_{k+2} \mid t_{k+1}} \rightarrow \cdots,
\end{aligned}
$$


where the notation $(\mathbf{x}, \mathbf{y})$ denotes the joint distribution of $\mathbf{x}$ and $\mathbf{y}$, and $\mathbf{x}_{t_{k+1} \mid t_{k+1}}$ denotes the distribution identical to that of the conditional distribution of $\mathbf{x}_{t_{k+1}}$ given the observations $\mathbf{y}_{t_{i}}, i \leq k+1$; by an abuse of notation, the same symbol may also denote a random variable having the aforementioned conditional distribution. If $u_{t_{k}}$ and $v_{t_{k}}$ are nearly linear functions, and the error terms are normally distributed, then the above conditional random variables may be well approximated by the normal random variables with means and covariance matrices computed by the UKF elaborated in the previous section. Specifically, let $\breve{\mathbf{x}}_{t_{k} \mid t_{k-1}}, \breve{\mathbf{x}}_{t_{k} \mid t_{k}}, \breve{\mathbf{y}}_{t_{k} \mid t_{k-1}}$ denote the approximating normal random variables with their means and covariance matrices computed from the UKF. First, we introduce some notations for keeping track of the propagation of the approximation error along the updating steps. Given $\breve{\mathbf{x}}_{t_{i} \mid t_{i}}$, define $\mathbf{x}_{\breve{\mathbf{x}}_{t_{i} \mid t_{i}}}\left(t_{k} \mid t_{k}\right)$ with $k \geq i$ as a random variable obtained as follows:

1. Starting with $\breve{\mathbf{x}}_{t_{i} \mid t_{i}}$, we obtain the conditional distribution of $\mathbf{x}$ at time $t_{i+1}$ given $\mathbf{Y}_{t_{i}}=\left\{\mathbf{y}_{t_{j}}, j \leq i\right\}$ to be denoted by $\mathbf{x}_{\breve{x}_{t_{i} \mid t_{i}}}\left(t_{i+1} \mid t_{i}\right)$ as follows:

$$
\mathbf{x}_{\breve{\mathbf{x}}_{t_{i} \mid t_{i}}}\left(t_{i+1} \mid t_{i}\right) \stackrel{d}{=} v_{t_{i}}\left(\breve{\mathbf{x}}_{t_{i} \mid t_{i}}\right)+\boldsymbol{\eta}_{t_{i}}
$$

where ' $\stackrel{d}{=}$ means 'equals in distribution.'

2. With $\mathbf{x}_{\breve{\mathbf{x}}_{t_{i} \mid t_{i}}}\left(t_{i+1} \mid t_{i}\right)$, we obtain the conditional distribution of $\mathbf{y}$ at time $t_{i+1}$ given $\mathbf{Y}_{t_{i}}$, to be denoted by $\mathbf{y}_{\mathbf{x}_{\breve{\mathbf{x}}_{t_{i} \mid t_{i}}}\left(t_{i+1} \mid t_{i}\right)}\left(t_{i+1} \mid t_{i}\right)$ as follows:

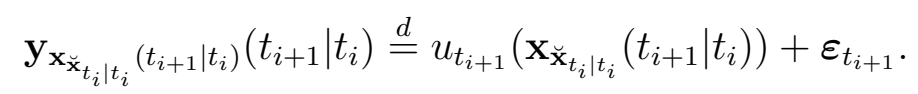

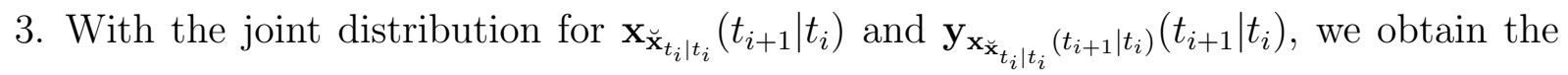
conditional distribution of $\mathbf{x}$ at time $t_{i+1}$ given $\mathbf{Y}_{t_{i+1}}$.

4. $i \leftarrow i+1$. Repeat 13 until $i=k$.

Thus, all conditional random variables from $\mathbf{x}_{\breve{\mathbf{x}}_{t_{i} \mid t_{i}}}\left(t_{i+1} \mid t_{i}\right)$ to $\mathbf{x}_{\breve{\mathbf{x}}_{t_{i} \mid t_{i}}}\left(t_{k} \mid t_{k}\right)$ are assumed to be obtained exactly from the model were the conditional distribution of $\mathbf{x}_{t_{i}}$ given $\mathbf{Y}_{t_{i}}$ identical to the distribution of $\breve{\mathbf{x}}_{t_{i} \mid t_{i}}$. In contrary, their true counterparts are denoted by, e.g. 
$\mathbf{x}_{\mathbf{x}_{t_{i} \mid t_{i}}}\left(t_{k} \mid t_{k}\right) \equiv \mathbf{x}\left(t_{k} \mid t_{k}\right)$ computed when the conditional distribution of $\mathbf{x}_{t_{i}}$ given $\mathbf{Y}_{t_{i}}$ is the true distribution from the model. Also we can define $\breve{\mathbf{x}}_{\breve{\mathbf{x}}_{t_{i} \mid t_{i}}}\left(t_{k} \mid t_{k}\right)$ as follows: starting with $\breve{\mathbf{x}}_{t_{i} \mid t_{i}}$, all conditional distributions from $\breve{\mathbf{x}}_{\breve{\mathbf{x}}_{t_{i} \mid t_{i}}}\left(t_{i+1} \mid t_{i}\right)$ to $\breve{\mathbf{x}}_{\breve{\mathbf{x}}_{t_{i} \mid t_{i}}}\left(t_{k} \mid t_{k}\right)$ are assumed to be conditional normal random variables with the approximate mean and covariance matrix obtained from the UKF. Thus, $\breve{\mathbf{x}}_{\breve{\mathbf{x}}_{t_{i} \mid t_{i}}}\left(t_{k} \mid t_{k}\right)=\breve{\mathbf{x}}_{t_{k} \mid t_{k}}$. Similar notations can be defined for the $\mathbf{y}$ process.

Below, we study the convergence properties of the UKF. First, we show that the UKF reduces to the Kalman filter for linear models.

Theorem 4. If the functions $u_{t_{k}}(\mathbf{x})$ and $v_{t_{k}}(\mathbf{x})$ in (3) are linear for $t_{0} \leq t_{k} \leq T, \varepsilon_{t_{k}} \sim$ $N\left(\mathbf{0}, \mathbf{H}_{t_{k}}\right)$, and $\boldsymbol{\eta}_{t_{k}} \sim N\left(\mathbf{0}, \mathbf{Q}_{t_{k}}\right)$, then we have

1. $\hat{\mathbf{x}}_{t_{k} \mid t_{k-1}}=E\left(\mathbf{x}_{t_{k} \mid t_{k-1}}\right), \hat{\mathbf{x}}_{t_{k} \mid t_{k}}=E\left(\hat{\mathbf{x}}_{t_{k} \mid t_{k}}\right), \hat{\mathbf{y}}_{t_{k} \mid t_{k-1}}=E\left(\mathbf{y}_{t_{k} \mid t_{k-1}}\right)$

2. $\hat{\mathbf{P}}_{\mathbf{x}, t_{k} \mid t_{k-1}}=\mathbf{P}_{\mathbf{x}, t_{k} \mid t_{k-1}}, \hat{\mathbf{P}}_{\mathbf{x}, t_{k} \mid t_{k}}=\mathbf{P}_{\mathbf{x}, t_{k} \mid t_{k}}, \hat{\mathbf{P}}_{\mathbf{y}, t_{k} \mid t_{k-1}}=\mathbf{P}_{\mathbf{y}, t_{k} \mid t_{k-1}}, \hat{\mathbf{P}}_{\mathbf{x y}, t_{k} \mid t_{k}}=\mathbf{P}_{\mathbf{x y}, t_{k} \mid t_{k}}$.

Proof. It follows from Theorem 2 in Section 4.

The following lemma of Milstein and Tretyakov (2004) is a key technical tool for studying the asymptotics of the UKF.

Lemma 1. Suppose that, for a fixed integer $N$ and $k=0, \ldots, N$, a sequence $\left\{u_{k}\right\}$ of nonnegative numbers satisfies the inequality

$$
u_{k+1} \leq(1+A h) u_{k}+B h^{p}
$$

where $h=T / N, A \geq 0, B \geq 0, p \geq 1$ are fixed constants. Then, for all $k=0, \ldots, N$,

$$
u_{k} \leq e^{A T} u_{0}+\frac{B}{A}\left(e^{A T}-1\right) h^{p-1}
$$

where for $A=0,\left(e^{A T}-1\right) / A$ is defined as zero.

Below, we state two general theorems quantifying the order of the UKF approximation error. For the UKF to be useful, the order of the approximation error should hold uniformly for all parameters over the parameter space, or at least over some compact neighborhood of the true parameter value. 
Theorem 5. Assume

1. $\left|E\left\{\mathbf{x}_{\breve{\mathbf{x}}_{t_{k} \mid t_{k}}}\left(t_{k+1} \mid t_{k+1}\right)-\breve{\mathbf{x}}_{\breve{\mathbf{x}}_{t_{k} \mid t_{k}}}\left(t_{k+1} \mid t_{k+1}\right)\right\}\right| \leq K\left(1+\left|E\left\{\breve{\mathbf{x}}_{t_{k} \mid t_{k}}\right\}\right|\right) h^{p}$,

2. $\left|E\left\{\mathbf{x}_{\breve{\mathbf{x}}_{t_{k} \mid t_{k}}}\left(t_{k+1} \mid t_{k+1}\right)-\breve{\mathbf{x}}_{t_{k} \mid t_{k}}\right\}\right| \leq K\left(1+\left|E\left\{\breve{\mathbf{x}}_{t_{k} \mid t_{k}}\right\}\right|\right) h$,

3. $\left|E\left\{\mathbf{x}_{\mathbf{x}_{t_{k} \mid t_{k}}}\left(t_{k+1} \mid t_{k+1}\right)-\mathbf{x}_{\breve{\mathbf{x}}_{t_{k} \mid t_{k}}}\left(t_{k+1} \mid t_{k+1}\right)\right\}\right| \leq\left|E\left\{\mathbf{x}_{t_{k} \mid t_{k}}-\breve{\mathbf{x}}_{t_{k} \mid t_{k}}\right\}\right|(1+K h)+K h^{p}$,

where $t_{k+1}-t_{k}=h=1 / s, t_{0} \leq t \leq T-h, p \geq 1$, and $h \leq 1$ and $K$ is uniformly bounded on the parameter space $\Theta$ and does not depend on $\mathbf{Y}_{T}=\left\{\mathbf{y}_{t_{0}}, \ldots, \mathbf{y}_{T}\right\}$. Then, for any $t_{k}$ between $t_{0}$ and $T-h$, the following inequality holds:

$$
\left|E\left\{\mathbf{x}_{t_{k} \mid t_{k}}-\breve{\mathbf{x}}_{t_{k} \mid t_{k}}\right\}\right| \leq K\left(1+\left|E\left\{\mathbf{x}_{0 \mid 0}\right\}\right|\right) h^{p-1}
$$

Before proving Theorem 5, we need the following lemma:

Lemma 2. Under Condition 1 and 2 of Theorem 5, for all positive integer $N$ and all $k=$ $0, \ldots, N$ the following inequality holds:

$$
\left|E\left\{\breve{\mathbf{x}}_{t_{k} \mid t_{k}}\right\}\right| \leq K\left(1+\left|E\left\{\mathbf{x}_{0 \mid 0}\right\}\right|\right)
$$

Proof. Clearly, we have the following equality:

$$
\begin{aligned}
\breve{\mathbf{x}}_{t_{k+1} \mid t_{k+1}}= & \breve{\mathbf{x}}_{t_{k} \mid t_{k}}+\left\{\mathbf{x}_{\breve{\mathbf{x}}_{t_{k} \mid t_{k}}}\left(t_{k+1} \mid t_{k+1}\right)-\breve{\mathbf{x}}_{t_{k} \mid t_{k}}\right\}-\left\{\mathbf{x}_{\breve{\mathbf{x}}_{t_{k} \mid t_{k}}}\left(t_{k+1} \mid t_{k+1}\right)-\breve{\mathbf{x}}_{t_{k+1} \mid t_{k+1}}\right\} \\
= & \breve{\mathbf{x}}_{t_{k} \mid t_{k}}+\left\{\mathbf{x}_{\breve{\mathbf{x}}_{t_{k} \mid t_{k}}}\left(t_{k+1} \mid t_{k+1}\right)-\breve{\mathbf{x}}_{t_{k} \mid t_{k}}\right\} \\
& -\left\{\mathbf{x}_{\breve{\mathbf{x}}_{t_{k} \mid t_{k}}}\left(t_{k+1} \mid t_{k+1}\right)-\breve{\mathbf{x}}_{\breve{\mathbf{x}}_{t_{k} \mid t_{k}}}\left(t_{k+1} \mid t_{k+1}\right)\right\}
\end{aligned}
$$

Using Condition 1 and 2 of Theorem 5 , we have

$$
\begin{aligned}
\left|\mathrm{E}\left\{\breve{\mathbf{x}}_{t_{k+1} \mid t_{k+1}}\right\}\right| \leq & \left|\mathrm{E}\left\{\breve{\mathbf{x}}_{t_{k} \mid t_{k}}\right\}\right|+\left|\mathrm{E}\left\{\mathbf{x}_{\breve{\mathbf{x}}_{t_{k} \mid t_{k}}}\left(t_{k+1} \mid t_{k+1}\right)-\breve{\mathbf{x}}_{t_{k} \mid t_{k}}\right\}\right| \\
& +\left|\mathrm{E}\left\{\mathbf{x}_{\breve{\mathbf{x}}_{t_{k} \mid t_{k}}}\left(t_{k+1} \mid t_{k+1}\right)-\breve{\mathbf{x}}_{\breve{\mathbf{x}}_{t_{k} \mid t_{k}}}\left(t_{k+1} \mid t_{k+1}\right)\right\}\right| \\
\leq & \left|\mathrm{E}\left\{\breve{\mathbf{x}}_{t_{k} \mid t_{k}}\right\}\right|+K\left(1+\left|\mathrm{E}\left\{\breve{\mathbf{x}}_{t_{k} \mid t_{k}}\right\}\right|\right) h+K\left(1+\left|\mathrm{E}\left\{\breve{\mathbf{x}}_{t_{k} \mid t_{k}}\right\}\right|\right) h^{p} \\
\leq & (1+K h)\left|\mathrm{E}\left\{\breve{\mathbf{x}}_{t_{k} \mid t_{k}}\right\}\right|+K h,
\end{aligned}
$$

where the last inequality holds because $p \geq 1$ and $h \leq 1$. Applying Lemma 1, we obtain the desired result. 
Proof of Theorem 5. First of all, we have the following equality:

$$
\begin{aligned}
\mathbf{x}_{t_{k+1} \mid t_{k+1}}-\breve{\mathbf{x}}_{t_{k+1} \mid t_{k+1}}= & \mathbf{x}_{\mathbf{x}_{t_{k} \mid t_{k}}}\left(t_{k+1} \mid t_{k+1}\right)-\mathbf{x}_{\breve{\mathbf{x}}_{t_{k} \mid t_{k}}}\left(t_{k+1} \mid t_{k+1}\right) \\
& +\mathbf{x}_{\breve{\mathbf{x}}_{t_{k} \mid t_{k}}}\left(t_{k+1} \mid t_{k+1}\right)-\breve{\mathbf{x}}_{\breve{\mathbf{x}}_{t_{k} \mid t_{k}}}\left(t_{k+1} \mid t_{k+1}\right) .
\end{aligned}
$$

Using Condition 1 and 3 of Theorem 5 , we obtain

$$
\begin{aligned}
\left|\mathrm{E}\left\{\mathbf{x}_{t_{k+1} \mid t_{k+1}}-\breve{\mathbf{x}}_{t_{k+1} \mid t_{k+1}}\right\}\right| \leq & \left|\mathrm{E}\left\{\mathbf{x}_{\mathbf{x}_{t_{k} \mid t_{k}}}\left(t_{k+1} \mid t_{k+1}\right)-\mathbf{x}_{\breve{\mathbf{x}}_{t_{k} \mid t_{k}}}\left(t_{k+1} \mid t_{k+1}\right)\right\}\right| \\
& +\left|\mathrm{E}\left\{\mathbf{x}_{\breve{\mathbf{x}}_{t_{k} \mid t_{k}}}\left(t_{k+1} \mid t_{k+1}\right)-\breve{\mathbf{x}}_{\breve{\mathbf{x}}_{t_{k} \mid t_{k}}}\left(t_{k+1} \mid t_{k+1}\right)\right\}\right| \\
\leq & \left|\mathrm{E}\left\{\mathbf{x}_{t_{k} \mid t_{k}}-\breve{\mathbf{x}}_{t_{k} \mid t_{k}}\right\}\right|(1+K h)+K\left(1+\left|\mathrm{E}\left\{\breve{\mathbf{x}}_{t_{k} \mid t_{k}}\right\}\right|\right) h^{p} \\
\leq & \left|\mathrm{E}\left\{\mathbf{x}_{t_{k} \mid t_{k}}-\breve{\mathbf{x}}_{t_{k} \mid t_{k}}\right\}\right|(1+K h)+K\left(1+\left|\mathrm{E}\left\{\mathbf{x}_{0 \mid 0}\right\}\right|\right) h^{p}
\end{aligned}
$$

where the last inequality follows from Lemma 2 . Using Lemma 1 and the fact that $\mathrm{E}\left\{\mathbf{x}_{0 \mid 0}-\right.$ $\left.\breve{\mathbf{x}}_{0 \mid 0}\right\}=\mathbf{0}$, we have

$$
\left|\mathrm{E}\left\{\mathbf{x}_{t_{k} \mid t_{k}}-\breve{\mathbf{x}}_{t_{k} \mid t_{k}}\right\}\right| \leq K\left(1+\left|\mathrm{E}\left\{\mathbf{x}_{0 \mid 0}\right\}\right|\right) h^{p-1}
$$

Theorem 5 indicates that in the case that $p>1$ with decreasing $h$, the average deviation between the $\mathbf{x}_{t_{k} \mid t_{k}}$ and its UKF approximation approaches 0 at the rate of $h^{p-1}$. Next, we extend Theorem 5 .

Theorem 6. Assume the conditions of Theorem 5 hold and that the followings hold

1. $\left|E\left\{\mathbf{y}_{\mathbf{x}_{t_{k} \mid t_{k}}}\left(t_{k+1} \mid t_{k}\right)-\mathbf{y}_{\breve{\mathbf{x}}_{t_{k} \mid t_{k}}}\left(t_{k+1} \mid t_{k}\right)\right\}\right| \leq K\left|E\left\{\mathbf{x}_{\mathbf{x}_{t_{k} \mid t_{k}}}\left(t_{k+1} \mid t_{k}\right)-\mathbf{x}_{\breve{\mathbf{x}}_{t_{k} \mid t_{k}}}\left(t_{k+1} \mid t_{k}\right)\right\}\right|+K h^{p-1}$,

2. $\left|E\left\{\mathbf{x}_{\mathbf{x}_{t_{k} \mid t_{k}}}\left(t_{k+1} \mid t_{k}\right)-\mathbf{x}_{\breve{\mathbf{x}}_{t_{k} \mid t_{k}}}\left(t_{k+1} \mid t_{k}\right)\right\}\right| \leq K\left(\left|E\left\{\mathbf{x}_{t_{k} \mid t_{k}}-\breve{\mathbf{x}}_{t_{k} \mid t_{k}}\right\}\right|\right)+K h^{p-1}$

3. $\left|E\left\{\mathbf{y}_{\breve{\mathbf{x}}_{t_{k} \mid t_{k}}}\left(t_{k+1} \mid t_{k}\right)-\breve{\mathbf{y}}_{\breve{\mathbf{x}}_{t_{k} \mid t_{k}}}\left(t_{k+1} \mid t_{k}\right)\right\}\right| \leq K\left|E\left\{\mathbf{x}_{\breve{\mathbf{x}}_{t_{k} \mid t_{k}}}\left(t_{k+1} \mid t_{k}\right)-\breve{\mathbf{x}}_{\breve{\mathbf{x}}_{t_{k} \mid t_{k}}}\left(t_{k+1} \mid t_{k}\right)\right\}\right|+K h^{p-1}$,

4. $\left|E\left\{\mathbf{x}_{\breve{\mathbf{x}}_{t_{k} \mid t_{k}}}\left(t_{k+1} \mid t_{k}\right)-\breve{\mathbf{x}}_{\breve{\mathbf{x}}_{t_{k} \mid t_{k}}}\left(t_{k+1} \mid t_{k}\right)\right\}\right| \leq K\left(1+\left|E\left\{\breve{\mathbf{x}}_{t_{k} \mid t_{k}}\right\}\right|\right) h^{p-1}$

where $K$ satisfies the condition required by Theorem 5. Then, for any $t_{k}$ between $t_{0}$ and $T-h$, we have

$$
\left|E\left\{\mathbf{y}_{t_{k+1} \mid t_{k}}-\breve{\mathbf{y}}_{t_{k+1} \mid t_{k}}\right\}\right| \leq K\left(1+\left|E\left(\mathbf{x}_{0 \mid 0}\right)\right|\right) h^{p-1}
$$


Proof.

$$
\begin{aligned}
\left|\mathrm{E}\left\{\mathbf{y}_{t_{k+1} \mid t_{k}}-\breve{\mathbf{y}}_{t_{k+1} \mid t_{k}}\right\}\right| \leq & \left|\mathrm{E}\left\{\mathbf{y}_{\mathbf{x}_{t_{k} \mid t_{k}}}\left(t_{k+1} \mid t_{k}\right)-\mathbf{y}_{\breve{\mathbf{x}}_{t_{k} \mid t_{k}}}\left(t_{k+1} \mid t_{k}\right)\right\}\right| \\
& +\left|\mathrm{E}\left\{\mathbf{y}_{\breve{\mathbf{x}}_{t_{k} \mid t_{k}}}\left(t_{k+1} \mid t_{k}\right)-\breve{\mathbf{y}}_{\breve{\mathbf{x}}_{t_{k} \mid t_{k}}}\left(t_{k+1} \mid t_{k}\right)\right\}\right| \\
\leq & K\left|\mathrm{E}\left\{\mathbf{x}_{\mathbf{x}_{t_{k} \mid t_{k}}}\left(t_{k+1} \mid t_{k}\right)-\mathbf{x}_{\breve{\mathbf{x}}_{t_{k} \mid t_{k}}}\left(t_{k+1} \mid t_{k}\right)\right\}\right| \\
& +K\left|\mathrm{E}\left\{\mathbf{x}_{\breve{\mathbf{x}}_{t_{k} \mid t_{k}}}\left(t_{k+1} \mid t_{k}\right)-\breve{\mathbf{x}}_{\breve{\mathbf{x}}_{t_{k} \mid t_{k}}}\left(t_{k+1} \mid t_{k}\right)\right\}\right|+K h^{p-1}
\end{aligned}
$$

(by Condition 1 and 3 )

$$
\leq K\left(\left|\mathrm{E}\left\{\mathbf{x}_{t_{k} \mid t_{k}}-\breve{\mathbf{x}}_{t_{k} \mid t_{k}}\right\}\right|\right)+K\left(1+\left|\mathrm{E}\left\{\breve{\mathbf{x}}_{t_{k} \mid t_{k}}\right\}\right|\right) h^{p-1}+K h^{p-1}
$$

(by Condition 2 and 4 )

$$
\leq K\left(1+\left|\mathrm{E}\left(\mathbf{x}_{0 \mid 0}\right)\right|\right) h^{p-1}
$$

(by Lemma 2 and Theorem 5).

Since $\left|\mathrm{E}\left\{\mathbf{y}\left(t_{k+1} \mid t_{k}\right)-\breve{\mathbf{y}}\left(t_{k+1} \mid t_{k}\right)\right\}\right|=O\left(h^{p-1}\right)$, we need $h^{p-1}<n^{-1}$, that is, $h<n^{-1 /(p-1)}$ to obtain consistency and asymptotical normality of our approximate CLS estimator via the UKF. In Section 6, we will outline an argument suggesting that the UKF can achieve an error rate up to $p=3$ for suitably discretized differential equation models. In contrast, the EKF can only achieve an error rate up to $p=2$ because the EKF uses only the first order term of the Taylor expansion. Thus, the UKF is more efficient than the EKF.

\section{LARGE-SAMPLE PROPERTIES OF THE UKF-CLS ESTIMATOR}

Often the state process is driven by some ODE or SDE. For ODEs, the state-space dynamics can be discretized via the Euler method or the fourth-order Runge-Kutta method (RK4), whereas for SDEs, the Euler method, the Milstein method, or the Runge-Kutta method may be employed for discretization, see Boyce and DiPrima (2004) and Milstein and Tretyakov (2004). The global truncation error rates of the Euler method and the RK4 for ODEs are $O(h)$ and $O\left(h^{4}\right)$, respectively. On the other hand, the strong orders of accuracy of the Euler method and the Milstein method for SDEs are 1/2 and 1, respectively, where a strong order 
$p$ for a discretized process $\left\{\hat{X}_{t_{k}}\right\}$ to a process $\left\{X_{t}\right\}$ with time step $t_{k+1}-t_{k} \equiv h$ is defined as follows:

$$
E\left|\hat{X}_{t_{k}}-X_{t_{k}}\right|=O\left(h^{p}\right)
$$

The Runge-Kutta methods for SDEs have various versions, of which strong orders can be greater than or equal to $3 / 2$. (We shall assume that the discretization error is of higher order than the error due to the use of the UKF scheme. This may place a restriction on which discretization method is permissible. For simplicity, we shall not pursue this issue.) They have the form as follows:

$$
\grave{\mathbf{x}}_{t_{k+1}}=\grave{\mathbf{x}}_{t_{k}}+h v_{t_{k}}\left(\grave{\mathbf{x}}_{t_{k}}\right)+\boldsymbol{\eta}_{t_{k}}, \boldsymbol{\eta}_{t_{k}} \sim\left(0, h \mathbf{Q}_{t_{k}}\right)
$$

where $\grave{\mathbf{x}}_{t_{k}}$ is the approximate solution at time $t_{k}$ and $t_{k+1}-t_{k}=h$. Note that the Euler method and the fourth-order Runge-Kutta method for an ODE do not have the error term, i.e., $\mathbf{Q}_{t_{k}}=\mathbf{0}$. Henceforth, we consider the following discretized state-space model:

$$
\begin{array}{rlrl}
\mathbf{y}_{t_{k}} & =u_{t_{k}}\left(\mathbf{x}_{t_{k}}\right)+\boldsymbol{\varepsilon}_{t_{k}}, & \boldsymbol{\varepsilon}_{t_{k}} \sim\left(0, \mathbf{H}_{t_{k}}\right), \\
\mathbf{x}_{t_{k+1}} & =\mathbf{x}_{t_{k}}+h v_{t_{k}}\left(\mathbf{x}_{t_{k}}\right)+\boldsymbol{\eta}_{t_{k}}, & \boldsymbol{\eta}_{t_{k}} \sim\left(0, h \mathbf{Q}_{t_{k}}\right), \\
\mathbf{x}_{0} & \sim\left(\mathrm{E}\left(\mathbf{x}_{0}\right), \mathbf{Q}_{0}\right) . & &
\end{array}
$$

Suppose $\breve{\mathbf{x}}_{t_{k} \mid t_{k}} \sim \mathrm{N}\left(\hat{\mathbf{x}}_{t_{k} \mid t_{k}}, h \mathbf{P}_{t_{k} \mid t_{k}}^{*}\right)$. Let $\breve{v}_{t_{k}}\left(\breve{\mathbf{x}}_{t_{k} \mid t_{k}}\right)$ be the normal approximation via the UKF corresponding to $v_{t_{k}}\left(\breve{\mathbf{x}}_{t_{k} \mid t_{k}}\right)$. Then,

$$
\begin{aligned}
\left|\mathrm{E}\left\{\mathbf{x}_{\breve{\mathbf{x}}_{t_{k} \mid t_{k}}}\left(t_{k+1} \mid t_{k}\right)-\breve{\mathbf{x}}_{\breve{\mathbf{x}}_{t_{k} \mid t_{k}}}\left(t_{k+1} \mid t_{k}\right)\right\}\right|= & \mid \mathrm{E}\left\{\breve{\mathbf{x}}_{t_{k} \mid t_{k}}+h v_{t_{k}}\left(\breve{\mathbf{x}}_{t_{k} \mid t_{k}}\right)\right\} \\
& -\mathrm{E}\left\{\breve{\mathbf{x}}_{t_{k} \mid t_{k}}+h \breve{v}_{t_{k}}\left(\breve{\mathbf{x}}_{t_{k} \mid t_{k}}\right)\right\} \mid \\
= & h\left|\mathrm{E}\left\{v_{t_{k}}\left(\breve{\mathbf{x}}_{t_{k} \mid t_{k}}\right)-\breve{v}_{t_{k}}\left(\breve{\mathbf{x}}_{t_{k} \mid t_{k}}\right)\right\}\right| \\
& =h^{3} \mathbf{d}_{k},
\end{aligned}
$$

for some vector $\mathbf{d}_{k}$ by Theorem 3 .

Here, we consider two scenarios: (i) $\mathbf{Q}_{t_{k}} \neq \mathbf{0}, 0 \equiv t_{0}<t_{k}<T$, for SDEs; (ii) $\mathbf{Q}_{t_{k}}=\mathbf{0}$, $0 \equiv t_{0}<t_{k}<T$, for ODEs. For each scenario, we assume that the $(i, j)$ th entry of $\mathbf{Q}_{0}$, denoted by $Q_{0, i j}$, satisfies the condition that $\left|Q_{0, i j}\right| \leq b K \leq h K$ for some $b \geq 0$ and $K \geq 0$, 
for all $i$ and $j$. We show that for all $k$, the covariance matrix of $\breve{\mathbf{x}}_{t_{k} \mid t_{k}}$ can be expressed as $h \mathbf{P}_{t_{k} \mid t_{k}}^{*}$ for some $\mathbf{P}_{t_{k} \mid t_{k}}^{*}$.

First of all, we show that the UT variance estimator of $\mathbf{y}=\mathbf{x}+h v(\mathbf{x})$ with $\mathbf{x} \sim(\mathrm{E}(\mathbf{x}), h \mathbf{P})$ can be written as $h \mathbf{P}^{*}$ for some $\mathbf{P}^{*}$. The sigma points are $\hat{\mathbf{x}}^{(0)}=\mathrm{E}(\mathbf{x}), \hat{\mathbf{x}}^{(i)}=\mathrm{E}(\mathbf{x})+$ $(\sqrt{(c+\lambda) h \mathbf{P}})_{i}^{T}, \hat{\mathbf{x}}^{(i+c)}=\mathrm{E}(\mathbf{x})-(\sqrt{(c+\lambda) h \mathbf{P}})_{i}^{T}$, for $i=1, \ldots, c$. Thus, the UT mean estimator $\hat{\mathbf{y}}=\mathrm{E}(\mathbf{x})+h \sum_{i=0}^{2 c} W^{(i)} v\left(\hat{\mathbf{x}}^{(i)}\right)$, which can be written as $\mathrm{E}(\mathbf{x})+h \mathbf{A}$ for some A. Hence $\hat{\mathbf{y}}^{(0)}-\hat{\mathbf{y}}=h v\{\mathrm{E}(\mathbf{x})\}-h \mathbf{A}, \hat{\mathbf{y}}^{(i)}-\hat{\mathbf{y}}=(\sqrt{(c+\lambda) h \mathbf{P}})_{i}^{T}+h v\left(\hat{\mathbf{x}}^{(i)}\right)-h \mathbf{A}$, and $\hat{\mathbf{y}}^{(i+c)}-\hat{\mathbf{y}}=-(\sqrt{(c+\lambda) h \mathbf{P}})_{i}^{T}+h v\left(\hat{\mathbf{x}}^{(i+c)}\right)-h \mathbf{A}, i=1, \ldots, c$. This implies that $\hat{\mathbf{P}}_{\mathbf{y}}=h \mathbf{P}^{*}$ for some $\mathbf{P}^{*}$.

Now we consider (i). Since $\left|Q_{0, i j}\right| \leq b K \leq h K$ for some $b \geq 0$ and $K \geq 0$, the variance of $\mathbf{x}_{0}$ can be expressed by $h \mathbf{Q}_{0}^{*}$ for some $\mathbf{Q}_{0}^{*}$. Thus, the variance of $\mathbf{x}_{t_{1} \mid t_{0}}, \hat{\mathbf{P}}_{\mathbf{x}, t_{1} \mid t_{0}}$ is $h \mathbf{P}_{t_{1} \mid t_{0}}^{*}$ for some $\mathbf{P}_{t_{1} \mid t_{0}}^{*}$ from the previous argument. If there is no observation at time $t_{1}$, we can show $\hat{\mathbf{P}}_{\mathbf{x}, t_{2} \mid t_{0}}=h \mathbf{P}_{\mathbf{x}, t_{2} \mid t_{0}}^{*}$ with proof similar to that of equation (14) in the Appendix of Ahn and Chan (2011). Note that $\hat{\mathbf{P}}_{\mathbf{x}, t_{2} \mid t_{0}}=\hat{\mathbf{P}}_{\mathbf{x}, t_{2} \mid t_{2}}$ in this case. In the presence of an observation at time $t_{1}$, it can be similarly shown that $\hat{\mathbf{P}}_{\mathbf{y}, t_{1} \mid t_{0}}=h \mathbf{R}_{\mathbf{y}, t_{1} \mid t_{0}}^{*}+\mathbf{H}_{t_{1}}$ for some $\mathbf{R}_{\mathbf{y}, t_{1} \mid t_{0}}^{*}$. Similarly, we obtain $\hat{\mathbf{P}}_{\mathbf{x y}, t_{1} \mid t_{0}}=h \mathbf{S}_{\mathbf{x y}, t_{1} \mid t_{0}}^{*}$ for some $\mathbf{S}_{\mathbf{x y}, t_{1} \mid t_{0}}^{*}$. Then, we have

$$
\begin{aligned}
\hat{\mathbf{P}}_{\mathbf{x}, t_{1} \mid t_{1}} & =\hat{\mathbf{P}}_{\mathbf{x}, t_{1} \mid t_{0}}-\hat{\mathbf{P}}_{\mathbf{x y}, t_{1} \mid t_{0}} \hat{\mathbf{P}}_{\mathbf{y}, t_{1} \mid t_{0}}^{-1} \hat{\mathbf{P}}_{\mathbf{x y}, t_{1} \mid t_{0}}^{T} \\
& =h \mathbf{Q}_{t_{1}}-h^{2} \mathbf{S}_{\mathbf{x y}, t_{1} \mid t_{0}}^{*} \hat{\mathbf{P}}_{\mathbf{y}, t_{1} \mid t_{0}}^{-1}\left(\mathbf{S}_{\mathbf{x y}, t_{1} \mid t_{0}}^{*}\right)^{T} \\
& \equiv h \hat{\mathbf{P}}_{\mathbf{x}, t_{1} \mid t_{1}}^{*} .
\end{aligned}
$$

By the same argument, $\hat{\mathbf{P}}_{\mathbf{x}, t_{2} \mid t_{1}}=h \mathbf{P}_{\mathbf{x}, t_{2} \mid t_{1}}^{*}$ for some $h \mathbf{P}_{\mathbf{x}, t_{2} \mid t_{1}}^{*}$ and $\hat{\mathbf{P}}_{\mathbf{x}, t_{2} \mid t_{2}}=h \hat{\mathbf{P}}_{\mathbf{x}, t_{2} \mid t_{2}}^{*}$ for some $\hat{\mathbf{P}}_{\mathbf{x}, t_{2} \mid t_{2}}^{*}$. By mathematical induction, $\hat{\mathbf{P}}_{\mathbf{x}, t_{k} \mid t_{k}}=h \hat{\mathbf{P}}_{\mathbf{x}, t_{k} \mid t_{k}}^{*}$ for all $k$. Thus, for the case of fixed initial state vector, we can obtain an error rate with $p=3$ for the UKF. Further, if $\mathbf{d}_{k}$ and $\hat{\mathbf{P}}_{\mathbf{x}, t_{k} \mid t_{k}}^{*}$ are uniformly bounded over the parameter space and the initial distribution, i.e., the distribution of $\mathbf{x}_{0}$, and if the conditions of Theorem 6 are satisfied, the UKF can achieve an error rate with $p=3$ or $O\left(h^{2}\right)$.

For (ii), using similar arguments as in case (i), $\hat{\mathbf{P}}_{\mathbf{x}, t_{2} \mid t_{1}}=h \mathbf{P}_{t_{2} \mid t_{1}}^{*}$ for some $\mathbf{P}_{t_{2} \mid t_{1}}^{*}$. By the same argument as in (i), we can show that $\hat{\mathbf{P}}_{\mathbf{x}, t_{k} \mid t_{k}}=h \hat{\mathbf{P}}_{\mathbf{x}, t_{k} \mid t_{k}}^{*}$ for all $k$. Thus, if $\mathbf{d}_{k}$ and 
$\hat{\mathbf{P}}_{\mathbf{x}, t_{k} \mid t_{k}}^{*}$ are uniformly bounded over the parameter space, and if the conditions of Theorem 6 are satisfied, the UKF can achieve an error rate with $p=3$ or $O\left(h^{2}\right)$. Thus, for an ODE, the entries of the covariance matrix of the initial state vector must be sufficiently small for the UKF to work.

If $h<n^{-1 /(p-1)}$, the approximate CLS estimator via the UKF enjoys the same consistency and asymptotic normality result of the exact CLS estimators. By similar argument, we can show that the EKF can achieve an error rate with $p=2$ because $\mathrm{E}(\mathbf{y})-\hat{\mathbf{y}}_{L}=O(h)$ as stated in Theorem 3. We formalize the preceding discussion by the following theorem:

Theorem 7. Consider the state-space model (5).

1. Assume that $\mathbf{Q}_{t_{k}} \neq \mathbf{0}$ for all $t_{k} \neq 0$. If the $\mathbf{d}_{k}$ and $\hat{\mathbf{P}}_{\mathbf{x}, t_{k} \mid t_{k}}^{*}$ are uniformly bounded, over the parameter space and for any initial state distribution, and if the conditions of Theorem [6 are satisfied, the UKF can achieve an error rate with $p=3$ or $O\left(h^{2}\right)$ and the EKF can achieve an error rate with $p=2$ or $O(h)$.

2. Assume that $\mathbf{Q}_{t_{k}}=\mathbf{0}$ for all $t_{k} \neq 0$. If the $\mathbf{d}_{k}$ and $\hat{\mathbf{P}}_{\mathbf{x}, t_{k} \mid t_{k}}^{*}$ are uniformly bounded over the parameter space, and if the conditions of Theorem 6 are satisfied, the UKF can achieve an error rate with $p=3$ or $O\left(h^{2}\right)$ and the EKF can achieve an error rate with $p=2$ or $O(h)$.

3. Moreover, if $h<n^{-1 /(p-1)}$ and condition (ii) of Theorem 1 holds, the approximate $C L S$ estimators via the UKF and EKF enjoy the same consistency and asymptotic normality result of the exact CLS estimators.

We remark that standard errors of the approximate CLS estimates can be computed by making use of the asymptotic normality result:

$$
\sqrt{n}\left(\hat{\boldsymbol{\theta}}-\boldsymbol{\theta}_{0}\right) \rightarrow_{d} \mathrm{~N}\left(0, \mathbf{V}^{-1} \mathbf{W} \mathbf{V}^{-1}\right),
$$

where $\mathbf{V}$ and $\mathbf{W}$ can be estimated with the partial derivatives approximated by finite differences, and confidence intervals of the parameters can then be constructed. 


\section{SIMULATION STUDY}

In this section, we report results from three simulation studies, with the first model satisfying the conditions of Theorem 1, 5, and 6. The other two simulation studies are based on an ODE system and a SDE system.

In Appendix, under assumptions (a)-(d) listed below, we verify the validity of the conditions stated in Theorems 5 and 6 for the following model:

$$
\begin{aligned}
\mathbf{y}_{t_{k}} & =\mathbf{Z}_{t_{k}} \mathbf{x}_{t_{k}}+\varepsilon_{t_{k}}, \\
\mathbf{x}_{t_{k+1}} & =\mathbf{x}_{t_{k}}+h g\left(\mathbf{x}_{t_{k}}\right)+\boldsymbol{\eta}_{t_{k}}
\end{aligned}
$$

where $\boldsymbol{\varepsilon}_{t_{k}} \sim\left(0, \mathbf{H}_{t_{k}}\right)$ and $\boldsymbol{\eta}_{t_{k}} \sim\left(\mathbf{0}, h \mathbf{Q}_{t_{k}}\right)$. It is assumed that $\mathbf{Z}_{t_{k}}$ is invertible for all $k$. Here, $\boldsymbol{\eta}_{t_{k}}$ may be a zero vector. Moreover, we assume

(a) $\left|\varepsilon_{t_{k}}\right| \leq K b^{p}$ with $0<b \leq h \leq 1$ and its covariance matrix $\mathbf{H}_{t_{k}}=c \mathbf{H}_{t_{k}}^{\prime}$ where $0<c \leq$ $K b^{p}$.

(b) $\left|\mathbf{y}_{t_{k}}\right|$ is bounded for all $k$.

(c) $g$ and $\left(\partial \mathbf{g}(\mathbf{x}) / \partial \mathbf{x}^{T}\right)_{i j}$ are bounded.

(d) $\left|\left(\partial^{2} \mathbf{g}(\mathbf{x}) / \partial \mathbf{x}^{T} \partial \mathbf{x}\right)_{i j}\right| \leq K h^{p-1}$ for all $i, j$.

\subsection{Example 1}

Consider the following model:

$$
\begin{aligned}
y_{t} & =x_{t}+\varepsilon_{t}, \\
\frac{d x_{t}}{d t} & =\theta x_{t}^{2}-x_{t}+\cos (0.5 t),
\end{aligned}
$$

where $\theta=0.1$ and $\varepsilon_{t} \sim \operatorname{Unif}(-0.1,0.1)$. The initial value is assumed to be known as $x_{0}=0$. To discretize the observation equation, we employed the RK4. For computing the "true" states, the RK4 with $h=1 / 30$ was employed. Observations were sampled for every 30 th state. The $y_{t}$ 's were generated by adding to the $x_{t}$ 's random errors distributed from 
True States and Sample Observations with $U(-0.1,0.1)$

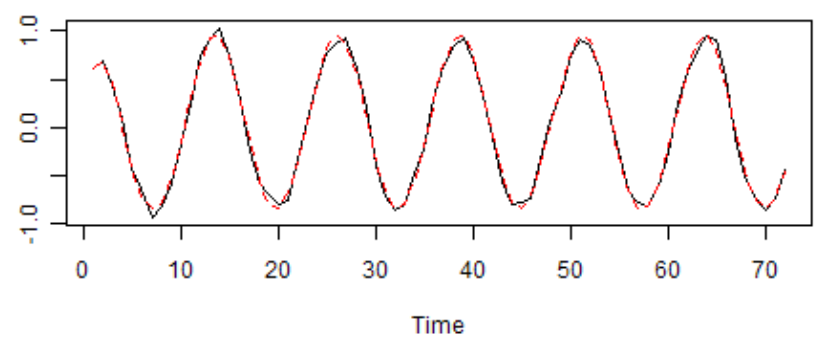

True States and Sample Observations with $\mathrm{N}(0,0.1)$

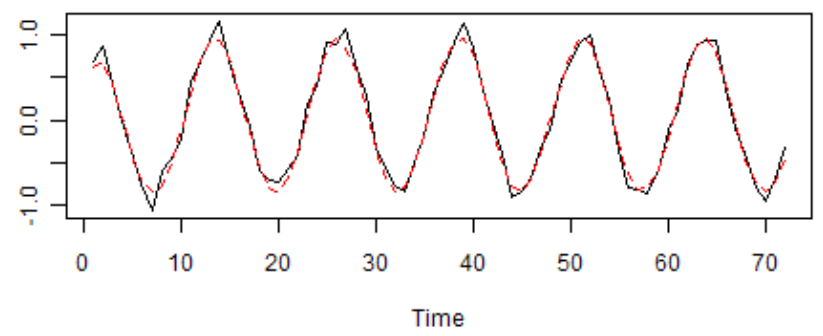

Figure 1: The upper graph shows true states (red dashed line) and the data (black solid line) simulated from the model with $\varepsilon \sim \operatorname{Unif}(-0.1,0.1)$. The lower graph shows another simulated realization from the model with $\varepsilon \sim \mathrm{N}(0,0.1)$. 
Unif $(-0.1,0.1)$. Let $f\left(t, x_{t}\right)=\theta x_{t}^{2}-x_{t}+\cos (0.5 t)$. The discretized state-space model is given by

$$
\begin{aligned}
y_{t_{k}} & =x_{t_{k}}+\varepsilon_{t_{k}}, \\
x_{t_{k+1}} & =x_{t_{k}}+\frac{h}{6}\left(k_{1, t_{k}}+2 k_{2, t_{k}}+2 k_{3, t_{k}}+k_{4, t_{k}}\right),
\end{aligned}
$$

where

$$
\begin{aligned}
& k_{1, t_{k}}=f\left(t_{k}, x_{t_{k}}\right), \\
& k_{2, t_{k}}=f\left(t_{k}+\frac{h}{2}, x_{t_{k}}+\frac{h}{2} k_{1, t_{k}}\right), \\
& k_{3, t_{k}}=f\left(t_{k}+\frac{h}{2}, x_{t_{k}}+\frac{h}{2} k_{2, t_{k}}\right), \\
& k_{4, t_{k}}=f\left(t_{k}+h, x_{t_{k}}+h k_{3, t_{k}}\right) .
\end{aligned}
$$

Let $g\left(x_{t_{k}}\right)=\left(k_{1, t_{k}}+2 k_{2, t_{k}}+2 k_{3, t_{k}}+k_{4, t_{k}}\right) / 6$. Conditions (a)-(b) stated in the beginning of this section can be shown to hold. We shall consider various step size $h$ with the smallest $h$ being $1 / 10$ and $K=100$. For simplicity, we estimate $\theta$ only. We use the 'optimize' function in $\mathrm{R}$ for minimizing the CLS objective function, with $\theta$ searched over the interval $(-10,10)$. Since we did not observe an initial value problems on optimization, we set it to 1 . We also have also repeated the experiment but with infinite-support noise, specifically, $\varepsilon_{t_{k}} \sim \mathrm{N}(0,0.1)$. Figure 7.1 shows the time plots of two simulated realizations. The experiment was replicated 1000 times. The step-size $h$ was chosen based on Condition 3 of Theorem 7 : $h<n^{-1 / 2}$. Table 1 shows the simulation results. As we can see in Table 1, as $h$ decreases with fixed $n=24$, the estimates become less biased although the standard deviation increases slightly. And as $n$ increases while $h$ is decreasing, the estimates are less biased and the standard deviation decreases. In addition, the estimates and standard deviations from the UKF are slightly less biased and less than those of the EKF, respectively. The empirical coverage rates of the 95\% confidence intervals, computed by the method discussed below Theorem 7, are generally close to 0.95 , but slightly less than 0.95 .

\subsection{Example 2}

The second simulation model is a variation of the SIR model. A typical SIR model assumes constant population size over time, see Diekmann and Heesterbeek (2000). We consider a 
Table 1: Simulation results for Example 1. The numbers in a row with row heading "M" are sample means, those with row heading "SD" are sample standard deviations and those with row heading "ECR" are the empirical coverage rate of the nominal 95\% confidence intervals.

\begin{tabular}{|c|c|c|c|c|c|}
\hline & & \multicolumn{2}{|c|}{$\operatorname{Unif}(-0.1,0.1)$} & \multicolumn{2}{|c|}{$\mathrm{N}(0,0.1)$} \\
\hline & & UKF & EKF & UKF & EKF \\
\hline \multirow[t]{3}{*}{$n=24, h=1$} & M & 0.09738 & 0.09724 & 0.09778 & 0.09758 \\
\hline & SD & 0.02752 & 0.02760 & 0.04837 & 0.04840 \\
\hline & ECR & 0.9419 & 0.9429 & 0.9410 & 0.9410 \\
\hline \multirow[t]{3}{*}{$n=24, h=1 / 2$} & M & 0.09874 & 0.09870 & 0.09904 & 0.09895 \\
\hline & SD & 0.02780 & 0.02787 & 0.04885 & 0.04888 \\
\hline & ECR & 0.9390 & 0.9410 & 0.9390 & 0.9400 \\
\hline \multirow[t]{3}{*}{$n=24, h=1 / 3$} & M & 0.09880 & 0.09877 & 0.09909 & 0.09901 \\
\hline & SD & 0.02780 & 0.02788 & 0.04887 & 0.04889 \\
\hline & ECR & 0.9390 & 0.9410 & 0.9390 & 0.9400 \\
\hline \multirow[t]{3}{*}{$n=24, h=1 / 5$} & M & 0.09881 & 0.09878 & 0.09910 & 0.09902 \\
\hline & SD & 0.02781 & 0.02788 & 0.04887 & 0.04889 \\
\hline & ECR & 0.9390 & 0.9410 & 0.9390 & 0.9400 \\
\hline \multirow[t]{3}{*}{$n=48, h=1 / 7$} & M & 0.10015 & 0.10013 & 0.09978 & 0.09975 \\
\hline & SD & 0.01816 & 0.01817 & 0.03158 & 0.03159 \\
\hline & ECR & 0.9495 & 0.9495 & 0.9343 & 0.9343 \\
\hline \multirow[t]{3}{*}{$n=72, h=1 / 10$} & M & 0.10006 & 0.10006 & 0.09961 & 0.09960 \\
\hline & SD & 0.01462 & 0.01463 & 0.02514 & 0.02515 \\
\hline & ECR & 0.9457 & 0.9467 & 0.9371 & 0.9381 \\
\hline
\end{tabular}



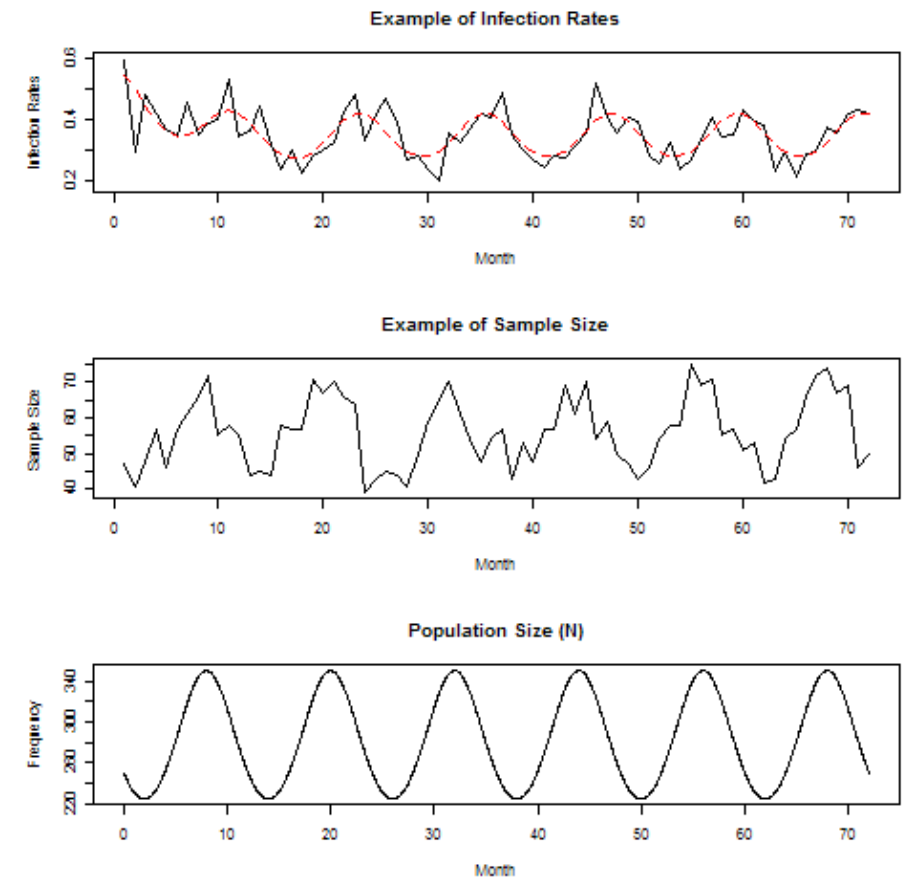

Figure 2: A simulated series: Red dashed line in the upper diagram shows the true infection rate. The solid line plots the observed infection rates based on the simulated trapping samples. The middle diagram plots the realized trapping size. The bottom diagram plots true population size. 
more general model which assumes that the population size changes overtime. Let $S, I, R$, be the sizes of susceptibles, infectives, recovered individuals, and $N$ the total population size, respectively. Also let $\alpha, \mu, \gamma$, and $b$ be the force of infection, death rate, recovery rate, and birth rate. The true (latent) state process satisfies the following system of differential equations:

$$
\begin{aligned}
\frac{d S}{d t} & =-\alpha \frac{S I}{N}+b N-\mu S, & \frac{d I}{d t} & =\alpha \frac{S I}{N}-\gamma I-\mu I, \\
\frac{d R}{d t} & =\gamma I-\mu R, & \frac{d N}{d t} & =-\mu N+b N,
\end{aligned}
$$

where $b=b_{t}=p \sin \left(\frac{\pi}{6} t\right)+q \cos \left(\frac{\pi}{6} t\right)+r$. Note that one of the equations in (7) is redundant because $S+I+R=N$. From Equation (7), we can obtain the following differential equations:

$$
\begin{aligned}
& \frac{d}{d t}\left(\frac{S}{N}\right)=-\alpha \frac{S}{N} \frac{I}{N}+\left(1-\frac{S}{N}\right) b, \\
& \frac{d}{d t}\left(\frac{I}{N}\right)=\alpha \frac{S}{N} \frac{I}{N}-(b+\gamma) \frac{I}{N},
\end{aligned}
$$

where $d / d t(R / N)$ was omitted because it is redundant. Equation $(8)$ is preferred to Equation (7) for estimation because it requires one less parameter as $\mu$ is dropped and its state space is the simplex so that the nonlinearity in Equation (8) may be less severe than Equation (7). From the full model (7), we simulated the population size $N_{t}$ and then generated the sample size $m_{t}$ from the binomial distribution $\operatorname{Bin}\left(N_{t}, a\right)$ where $a$ is the capture rate. Observations are taken at time $t=1,2, \ldots, n$; at time $t, y_{t}$ is drawn from the binomial distribution $\operatorname{Bin}\left(m_{t}, I_{t} / N_{t}\right)$, with $y_{t} / m_{t}$ being the observed sample proportion of infectives. The $y$ 's are conditionally independent given $m_{t}, I_{t} / N_{t}, t=1,2, \ldots, n$. The true parameter vector $(\alpha, \mu, \gamma, p, q, r)=(1,0.15,0.15,0.06,-0.1,0.15)$, with the initial proportion of susceptibles being $s_{0}=0.25$ and that of infectives equal to $i_{0}=0.55$. The fourth-order Runge-Kutta method was employed to generate the underlying continuous-time process. The discretization step size is set to $h=1 / 30$ corresponding to 1 day, whereas the proportions of infectives were measured as the sample proportion of infective subjects, once per month.

Each simulated series is 72 months long, hence there are $72 \times 30=2160$ state vectors. Figure 2 shows a simulated series of observations. For statistical analysis, the UKF-CLS 
method was applied to fit the following state-space model:

$$
\begin{aligned}
\frac{y_{t_{k}}}{m_{t}} & =\frac{I_{t_{k}}}{N_{t_{k}}}+\varepsilon_{t_{k}}, \varepsilon_{t_{k}} \sim \mathrm{N}\left(0, \frac{y_{t_{k}}\left(1-y_{t_{k}}\right)}{m_{t_{k}}}\right), \\
\mathbf{x}_{t_{k+1}} & =\mathbf{x}_{t_{k}}+\frac{h}{6}\left(k_{1, t_{k}}+2 k_{2, t_{k}}+2 k_{3, t_{k}}+k_{4, t_{k}}\right),
\end{aligned}
$$

where $t_{k+k}=t_{k}+h$ for some $h>0$, and $\mathbf{x}_{t_{k+1}}$ is the fourth-order Runge-Kutta discretization of the latent state process $\left\{x(t)=\left(S_{t} / N_{t}, I_{t} / N_{t}\right)^{T}\right\}$ driven by the differential equation

$$
\begin{aligned}
& \frac{d}{d t}\left(\frac{S}{N}\right)=-\alpha \frac{S}{N} \frac{I}{N}+\left(1-\frac{S}{N}\right) b, \\
& \frac{d}{d t}\left(\frac{I}{N}\right)=\alpha \frac{S}{N} \frac{I}{N}-(b+\gamma) \frac{I}{N},
\end{aligned}
$$

where

$$
b=b_{t}=p \sin \left(\frac{\pi}{6} t\right)+q \cos \left(\frac{\pi}{6} t\right)+r .
$$

The variance of $\varepsilon_{t_{k}}$ was obtained from the variance of the binomial distribution. Optimization of the approximate CLS was done in $\mathrm{R}$ with the "optim" function. In order to apply the UKF, we need to specify the distribution of the initial state vector which is taken to be some distribution with mean vector $\left(s_{0}, i_{0}\right)$ and covariance matrix given by

$$
\frac{1}{N_{0}}\left(\begin{array}{cc}
s_{0}\left(1-s_{0}\right) & -s_{0} i_{0} \\
-s_{0} i_{0} & i_{0}\left(1-i_{0}\right)
\end{array}\right),
$$

where $N_{0}$ is the initial population size; this specification is motivated by the fact that $\left(S_{0}, I_{0}, R_{0}\right)$ has a multinomial distribution $\operatorname{Multinom}\left(N_{0},\left(s_{0}, i_{0}, r_{0}\right)\right)$. In practice, $s_{0}, i_{0}$ and $N_{0}$ are unknown, and they could be estimated by the UKF-CLS, by augmenting them into the parameter vector. The initial population size $N_{0}$ is fixed at 150 (the true $N_{0}$ is 250) in the simulation study because empirically $N_{0}$ shows little impact on the other estimated parameter values.

For the UKF computations, we set $\lambda$ of Section 3 to zero for simplicity. The starting values for $\left(\alpha, \mu, \gamma, p, q, r, s_{0}, i_{0}\right)$ in optimization are generated from the uniform distributions: Unif(0.6, 1.4), Unif(0.05, 0.25), Unif(0, 0.12), Unif(-0.2, 0), Unif(0.05, 0.25), Unif(0.10, 0.40), and Unif $(0.40,0.70)$, respectively. The trapping proportion $a$ is set to 0.2 , i.e., about $20 \%$ 
Table 2: Simulation results for estimation equation (8). The numbers in a row with row heading "M" are sample means and those with row heading "SD" are sample standard deviations

\begin{tabular}{|c|c|c|c|c|c|c|c|c|}
\hline$h \& n$ & & $\begin{array}{l}\alpha \\
(1) \\
\end{array}$ & $\begin{array}{c}\gamma \\
(0.15) \\
\end{array}$ & $\begin{array}{c}p \\
(0.06) \\
\end{array}$ & $\begin{array}{c}q \\
(-0.1) \\
\end{array}$ & $\begin{array}{c}r \\
(0.15) \\
\end{array}$ & $\begin{array}{c}i_{0} \\
(0.25) \\
\end{array}$ & $\begin{array}{c}s_{0} \\
(0.55) \\
\end{array}$ \\
\hline$h=1$ & $\mathrm{M}$ & 1.044 & 0.143 & 0.058 & -0.085 & 0.168 & 0.263 & 0.564 \\
\hline$n=24$ & $\mathrm{SD}$ & 0.415 & 0.039 & 0.056 & 0.049 & 0.066 & 0.134 & 0.116 \\
\hline$h=1 / 5$ & $\mathrm{M}$ & 1.046 & 0.142 & 0.057 & -0.084 & 0.167 & 0.266 & 0.561 \\
\hline$n=24$ & $\mathrm{SD}$ & 0.414 & 0.038 & 0.056 & 0.048 & 0.064 & 0.134 & 0.110 \\
\hline$h=1$ & M & 1.025 & 0.143 & 0.060 & -0.087 & 0.166 & 0.261 & 0.560 \\
\hline$n=48$ & $\mathrm{SD}$ & 0.343 & 0.035 & 0.047 & 0.038 & 0.056 & 0.119 & 0.100 \\
\hline$h=1$ & M & 1.008 & 0.142 & 0.061 & -0.087 & 0.165 & 0.257 & 0.561 \\
\hline$n=72$ & $\mathrm{SD}$ & 0.320 & 0.034 & 0.044 & 0.035 & 0.055 & 0.105 & 0.093 \\
\hline
\end{tabular}

of the subjects are trapped at the end of each month in order to compute the proportion of infectives. Since we found that the RK4 with $h=1,1 / 2,1 / 3,1 / 5,1 / 15,1 / 30$ produces almost identical state vector values and the UKF also shows very similar estimates, we ran $n=24$ with $h=1,1 / 5, n=48$ with $h=1$, and $n=72$ with $h=1$. Each experiment was replicated 1000 times. Table 2 summarizes the simulation results. The numbers in parenthesis in the first row of the table are the true parameter values. The results of $n=24$ with $h=1$ and $n=24$ with $h=1 / 5$ are very similar. On average, all parameter estimators are close to the true parameters. Moreover, as $n$ increases, the estimates become slightly closer to the true parameters, on average. Furthermore, the sample standard deviations decrease with increasing sample size. 

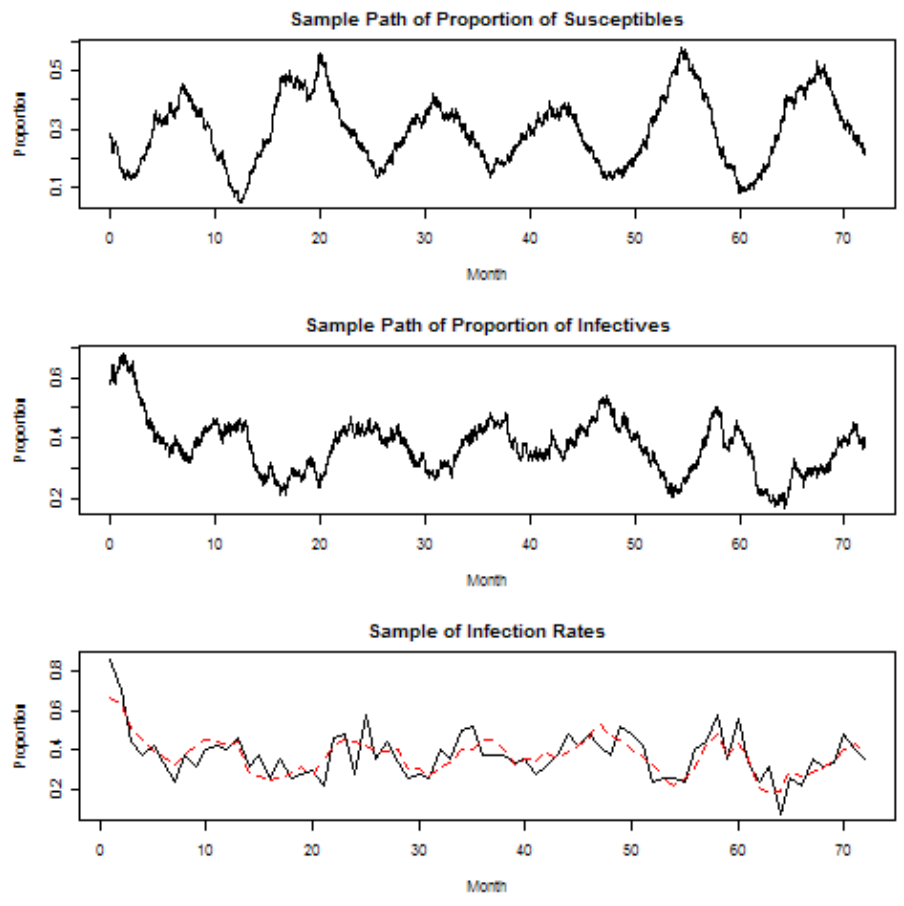

Figure 3: A simulated series: The first two upper diagrams plot the sample paths of $s_{t}$ and $i_{t}$. In the bottom diagram, the solid line plots the observed infection rates based on the simulated trapping samples. The red dashed line shows the true infection rate. 


\subsection{Example 3}

In this section, we consider an example of the SDE, which is a stochastic version of (8):

$$
\left(\begin{array}{c}
d s_{t} \\
d i_{t}
\end{array}\right)=\left(\begin{array}{c}
-\alpha s_{t} i_{t}+\left(1-s_{t}\right) b_{t} \\
\alpha s_{t} i_{t}-\left(b_{t}+\gamma\right) i_{t}
\end{array}\right) d t+k \mathbf{B}_{t}\left(\begin{array}{c}
d w_{1}(t) \\
d w_{2}(t)
\end{array}\right),
$$

where $s_{t}$ and $i_{t}$ represent the proportion of susceptibles and infectives, and $w_{i}(t)$ are two independent standard Brownian motion for $i=1,2$ with

$$
\mathbf{B}_{t} \mathbf{B}_{t}^{\prime}=\left(\begin{array}{cc}
s_{t}\left(1-s_{t}\right) & -s_{t} i_{t} \\
-s_{t} i_{t} & i_{t}\left(1-i_{t}\right)
\end{array}\right)
$$

The state equation may be discretized by several methods, e.g. the Euler's method, Milstein's

method, and Runge-Kutta method, see Milstein and Tretyakov (2004). We employ the Euler's method. The discretized state equation is

$$
\left(\begin{array}{c}
s_{t_{k+1}} \\
i_{t_{k+1}}
\end{array}\right)=\left(\begin{array}{c}
s_{t_{k}} \\
i_{t_{k}}
\end{array}\right)+\left(\begin{array}{c}
-\alpha s_{t_{k}} i_{t_{k}}+\left(1-s_{t_{k}}\right) b_{t_{k}} \\
\alpha s_{t_{k}} i_{t_{k}}-\left(b_{t_{k}}+\gamma\right) s_{t_{k}}
\end{array}\right) h+k \mathbf{B}_{t_{k}}\left(\begin{array}{c}
\Delta w_{1}\left(t_{k}\right) \\
\Delta w_{2}\left(t_{k}\right)
\end{array}\right),
$$

where $\Delta w_{i}\left(t_{k}\right)=w_{i}\left(t_{k+1}\right)-w_{i}\left(t_{k}\right)$ for $i=1,2$. Since $w_{i}\left(t_{k}\right)$ indicates the standard Brownian motion, the state-space model becomes

$$
\begin{aligned}
y_{t} & =i_{t}+\varepsilon_{t}, \quad \varepsilon_{t} \sim \mathrm{N}\left(0, \frac{y_{t}\left(1-y_{t}\right)}{m_{t}}\right), \\
\left(\begin{array}{c}
s_{t_{k+1}} \\
i_{t_{k+1}}
\end{array}\right) & =\left(\begin{array}{c}
s_{t_{k}} \\
i_{t_{k}}
\end{array}\right)+\left(\begin{array}{c}
-\alpha s_{t_{k}} i_{t_{k}}+\left(1-s_{t_{k}}\right) b_{t_{k}} \\
\alpha s_{t_{k}} i_{t_{k}}-\left(b_{t_{k}}+\gamma\right) s_{t_{k}}
\end{array}\right) h+k \mathbf{B}_{t_{k}} \boldsymbol{\eta}_{t_{k}},
\end{aligned}
$$

where

$$
\begin{aligned}
b_{t_{k}} & =p \sin \left(\frac{\pi}{6} t_{k}\right)+q \cos \left(\frac{\pi}{6} t_{k}\right)+r, \\
\boldsymbol{\eta}_{t_{k}} & \sim \mathrm{N}\left(\mathbf{0},\left(\begin{array}{cc}
h & 0 \\
0 & h
\end{array}\right)\right), \\
\mathbf{B}_{t_{k}} \mathbf{B}_{t_{k}}^{\prime} & =\left(\begin{array}{cc}
s_{t_{k}}\left(1-s_{t_{k}}\right) & -s_{t_{k}} i_{t_{k}} \\
-s_{t_{k}} i_{t_{k}} & i_{t_{k}}\left(1-i_{t_{k}}\right)
\end{array}\right) .
\end{aligned}
$$

The distribution of the initial state vector is

$$
\mathrm{N}\left(\left(\begin{array}{c}
s_{0} \\
i_{0}
\end{array}\right), k^{2}\left(\begin{array}{cc}
s_{0}\left(1-s_{0}\right) & -s_{0} i_{0} \\
-s_{0} i_{0} & i_{0}\left(1-i_{0}\right)
\end{array}\right)\right) .
$$


Table 3: Simulation results for the SDE model. The numbers in a row with row heading "M" are sample means and those with row heading "SD" are sample standard deviations

\begin{tabular}{|c|c|c|c|c|c|c|c|c|c|}
\hline$h \& n$ & & $\begin{array}{l}\alpha \\
(1)\end{array}$ & $\begin{array}{c}\gamma \\
(0.15)\end{array}$ & $\begin{array}{c}p \\
(0.06)\end{array}$ & $\begin{array}{c}q \\
(-0.1)\end{array}$ & $\begin{array}{c}r \\
(0.15)\end{array}$ & $\begin{array}{c}i_{0} \\
(0.25)\end{array}$ & $\begin{array}{c}s_{0} \\
(0.55)\end{array}$ & $\begin{array}{c}k \\
(0.01)\end{array}$ \\
\hline$h=1$ & M & 1.052 & 0.142 & 0.054 & -0.050 & 0.162 & 0.301 & 0.531 & 0.011 \\
\hline$n=24$ & $\mathrm{SD}$ & 0.363 & 0.034 & 0.038 & 0.034 & 0.062 & 0.137 & 0.115 & 0.008 \\
\hline$h=1 / 2$ & M & 1.066 & 0.146 & 0.056 & -0.070 & 0.169 & 0.297 & 0.543 & 0.011 \\
\hline$n=24$ & $\mathrm{SD}$ & 0.404 & 0.037 & 0.046 & 0.042 & 0.064 & 0.144 & 0.120 & 0.007 \\
\hline$h=1 / 3$ & M & 1.075 & 0.147 & 0.057 & -0.076 & 0.172 & 0.294 & 0.546 & 0.011 \\
\hline$n=24$ & $\mathrm{SD}$ & 0.420 & 0.038 & 0.051 & 0.045 & 0.066 & 0.147 & 0.120 & 0.008 \\
\hline$h=1 / 5$ & M & 1.069 & 0.148 & 0.056 & -0.082 & 0.173 & 0.290 & 0.549 & 0.011 \\
\hline$n=24$ & $\mathrm{SD}$ & 0.388 & 0.038 & 0.054 & 0.046 & 0.066 & 0.142 & 0.119 & 0.008 \\
\hline$n=48$ & M & 1.054 & 0.149 & 0.057 & -0.087 & 0.170 & 0.275 & 0.557 & 0.011 \\
\hline$h=1 / 7$ & $\mathrm{SD}$ & 0.338 & 0.036 & 0.045 & 0.037 & 0.057 & 0.128 & 0.106 & 0.008 \\
\hline$n=72$ & $\mathrm{M}$ & 1.046 & 0.149 & 0.059 & -0.087 & 0.170 & 0.263 & 0.560 & 0.012 \\
\hline$h=1 / 10$ & $\mathrm{SD}$ & 0.325 & 0.035 & 0.043 & 0.034 & 0.054 & 0.116 & 0.094 & 0.008 \\
\hline
\end{tabular}

The experiment was replicated 1000 times. Since the Euler method is less accurate than the Milstein's method and the Runge-Kutta method, the sample path of the true states were generated using the Euler method with $h=1 / 60$, with observations presumed to be measured every 60 th true state. We fixed the sample size $m_{t}=50$ and generated the sample infection proportions from $\operatorname{Bin}\left(50, i_{t_{k}}\right)$. Figure 3 shows the time plots of a realization. We used the same starting values as described in Example 2 for optimization. Table 3 summarizes the simulation results. The results of $n=24$ with $h=1,1 / 2,1 / 3,1 / 5$ show that in general as $h$ decreases, the estimates become closer to the true parameters, on average. Further, as $n$ increases and $h$ decreases, the estimates become slightly closer to the true parameters, on 

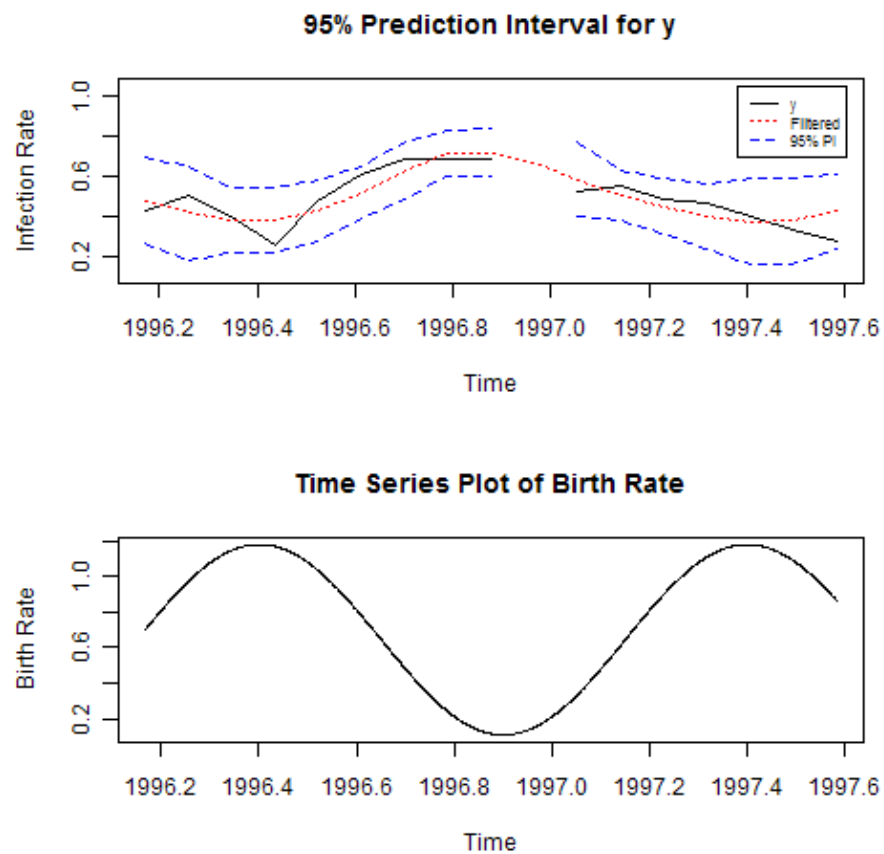

Figure 4: Upper diagram plots the observed and fitted values. Lower diagram plots the estimated birth rate function.

average, with smaller sample standard deviations.

\section{REAL EXAMPLE: PREVALENCE OF BARTONELLA INFECTION IN A WILD POPULATION OF COTTON RATS}

We analyze a dataset from a monitoring study, conducted by CDC, on the prevalence of bartonella infection in a wild cotton rat population over a period of 17 months, from March, 1996 to July, 1997 (Kosoy, Mandel, Green, Marston, Jones and Childs 2004; Chan and Kosoy

Table 4: Estimates and standard errors of the fitted SIR model.

\begin{tabular}{cccccccc}
\hline & $\alpha$ & $\gamma$ & $p$ & $q$ & $r$ & $s_{0}$ & $i_{0}$ \\
\hline$\hat{\boldsymbol{\theta}}$ & 2.127 & 0.109 & 0.534 & 0.057 & 0.645 & 0.318 & 0.552 \\
s.e. & 0.485 & 0.018 & 0.149 & 0.048 & 0.137 & 0.113 & 0.103 \\
\hline
\end{tabular}


2010). But trapping was not done in December, 1996, resulting in a lone missing data case. So there were 16 observed bartonella (monthly) infection rates, with all bartonella variants included. In June and July in 1996, four cotton rats gave birth in their traps. To avoid issues related to vertical transmission of bartonella infection from parent subjects to their children, we excluded 10 rats in June, 1996 (Rat \#: $36_{1}, \ldots, 36_{6}$ and $1_{1}, \ldots, 1_{4}$ ) and 9 rats in July, 1996 (Rat \#: 104 $1, \ldots, 104_{5}$ and $109_{1}, \ldots, 109_{4}$ ). We shall analyze the data based on the SIR framework specified by Equation (8). As explained earlier, for estimation purpose, Equation (8) is preferred so that all results reported below is based on Equation (8) fitted by the proposed UKF-CLS method. We fixed $N_{0}=150$ and $h=1$. In the upper diagram of Figure 4, the solid black line plots the sample infection rates and the red dotted line plots the predicted values based on the past data, that is, $\hat{\mathrm{E}}\left(i_{t} \mid y_{1}, \cdots, y_{t-1}, m_{1}, \ldots, m_{t-1}\right)$, computed from the fitted SIR model. The blue dotted line indicates the nominal 95\% prediction interval assuming the predictors are normally distributed with the prediction variance obtained from the UKF. All observations are inside the $95 \%$ prediction intervals. The estimated birth rate curve (lower diagram of Figure 4) shows that as the birth rate increases, the infection rate decreases and vice versa, which matches general epidemiological considerations. Table 4 shows the estimated parameter values and their standard errors, based on the asymptotic distribution results for the UKF-CLS estimator, see the discussion below Theorem 7. The estimated values are $\hat{\alpha}=2.127, \hat{\gamma}=0.109, \hat{p}=0.534, \hat{q}=0.057, \hat{r}=0.645, \hat{s}_{0}=0.318$, and $\hat{i}_{0}=0.552$. Note that $\left(\hat{s}_{0}, \hat{i}_{0}\right)$ is the estimated value for one month before March, 1996, that is, the estimated value for February, 1996. For interpretation, the mean monthly recovery rate of infectives is $0.109(\hat{\gamma})$, i.e., $10.9 \%$ of infectives are recovered a month after infection on average. The estimate $\hat{\alpha}=2.127$ is the product of the transmission probability and the number of contacts. In other words, the average number of new infections per infected subject per month is 2.127 . The birth rate $b$ indicates the birth rate per capita, which is estimated to attain the maximum in May and the mimimum in November.

We now assess the goodness of fit of the fitted SIR model, by checking whether or not the standardized residuals are approximately independent and identically distributed. The 

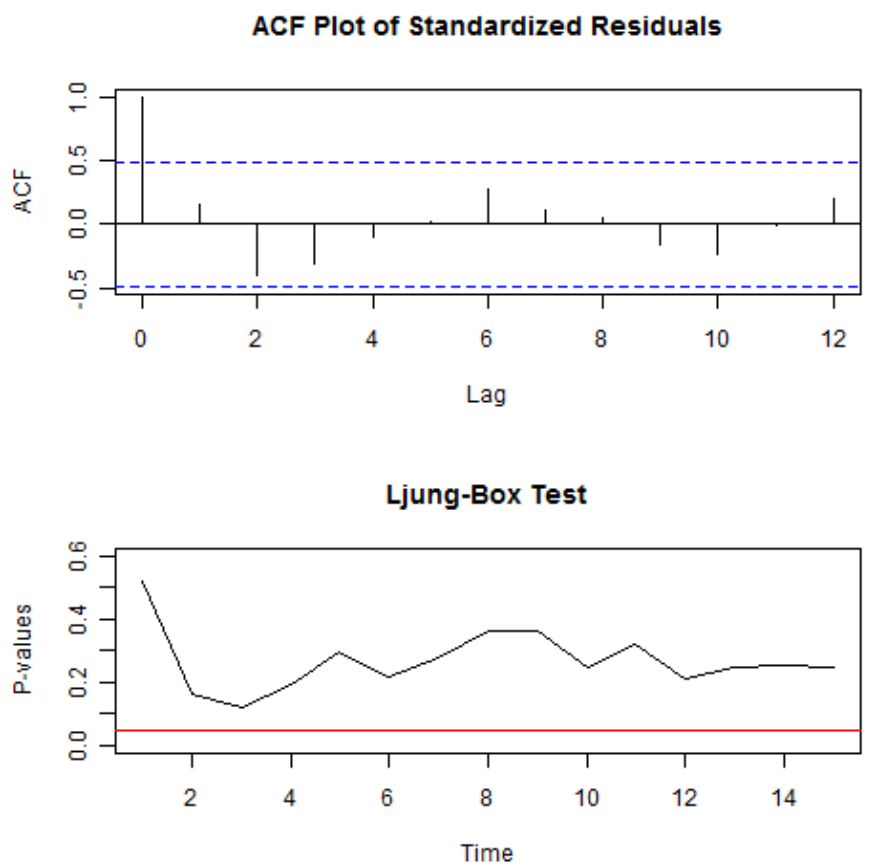

Figure 5: Model diagnostics of the SIR model fitted to the cotton rat data. Upper figure plots the residual autocorrelation function which suggests that the standardized residuals are not serially correlated. This is confirmed by the plot of the p-values of the Box-Ljung test for no residual autocorrelations based on cumulative information of the first $k$ lags of residual autocorrelations, for $k=1,2, \cdots, 15$. The horizontal line indicates the $5 \%$ level. 
residuals are defined by the observed values minus the predicted values which are generally the 1-step ahead predictors. The residuals can then be standardized by normalizing them by the predicted standard deviations. Figure 5 shows the ACF plot and the p-values of the Ljung-Box plot of standardized residuals which are useful for checking the iid assumption of the standardized residuals. The ACF plot and the Ljung-Box plot show that the residuals appear to be white noise, suggesting that the model fits the data well.

\section{DISCUSSION}

A new approach for implementing the method of conditional least squares via UKF has been proposed for estimating a continuous-time process with discrete-time data. Simulation studies confirm that the UKF-CLS method enjoys good empirical performance. In addition, the new model describes the dynamics of the real data example well.

The UKF has several advantages over the EKF and simulation based methods. First, the UKF does not need to calculate the Jacobian matrix, which may not be available. Second, the UKF uses a deterministic scheme to choose the sigma points. This makes the UKF computationally more efficient than simulation based methods. For the case of the discretized state-space model of a process defined by some differential equation, the 1-step ahead predictor can be computed by the UKF with an error of $O\left(h^{2}\right)$ which is better than the EKF.

Moreover, the faster convergence rate leads to the consistency and asymptotic normality of the UKF-CLS estimator with sufficiently small step-size $h$ which is less than $n^{-1 /(p-1)}$. An interesting statistical problem is to generalize the proposed approach to Gaussian-likelihood estimation.

\section{ACKNOWLEDGEMENTS}

This work was partially supported by the U.S. National Science Foundation. The authors would like to thank Dr. Michael Kosoy for providing us the Bartonella infection data for the real analysis. 


\section{APPENDIX A. PROOF OF EXAMPLE IN SECTION 7}

We give the proof that the example in the beginning of Section 7 satisfies the conditions of Theorems 5 and 6 .

There are two cases: i) We have an observation at time $t_{k+1}$; ii) We do not have an observation at time $t_{k+1}$.

- i) We observe $\mathbf{y}_{t_{k+1}}$.

- Let's consider Condition 1 of Theorem 5. Since

$$
\mathbf{y}_{t_{k}}=\mathbf{Z}_{t_{k}} \mathbf{x}_{t_{k}}+\varepsilon_{t_{k}}
$$

we have

$$
\begin{aligned}
& \mathrm{E}\left\{\mathbf{x}_{\breve{\mathbf{x}}_{t_{k} \mid t_{k}}}\left(t_{k+1} \mid t_{k+1}\right)\right\} \\
& =\mathrm{E}\left\{\mathbf{Z}_{t_{k}}^{-1} \mathbf{y}_{\breve{\mathbf{x}}_{t_{k} \mid t_{k}}}\left(t_{k+1} \mid t_{k+1}\right)\right\}-\mathrm{E}\left\{\mathbf{Z}_{t_{k}}^{-1} \varepsilon_{\breve{\mathbf{x}}_{t_{k} \mid t_{k}}}\left(t_{k+1} \mid t_{k+1}\right)\right\} \\
& =\mathbf{Z}_{t_{k}}^{-1} \mathbf{y}_{t_{k+1}}-\mathbf{Z}_{t_{k}}^{-1} \mathrm{E}\left\{\boldsymbol{\varepsilon}_{\breve{\mathbf{x}}_{t_{k} \mid t_{k}}}\left(t_{k+1} \mid t_{k+1}\right)\right\} .
\end{aligned}
$$

Let $\mathbf{M}_{t_{k}}=\hat{\mathbf{P}}_{\mathbf{x}, t_{k+1} \mid t_{k}} \mathbf{Z}_{t_{k}}^{-1}$ and $\mathbf{F}_{t_{k}}=\mathbf{Z}_{t_{k}} \hat{\mathbf{P}}_{\mathbf{x}, t_{k+1} \mid t_{k}} \mathbf{Z}_{t_{k}}^{-1}+\mathbf{H}_{t_{k}}$. By linear projection, we have

$$
\begin{aligned}
& \mathrm{E}\left\{\breve{\mathbf{x}}_{\breve{\mathbf{x}}_{t_{k} \mid t_{k}}}\left(t_{k+1} \mid t_{k+1}\right)\right\} \\
& =\mathrm{E}\left\{\breve{\mathbf{x}}_{\breve{\mathbf{x}}_{t_{k} \mid t_{k}}}\left(t_{k+1} \mid t_{k}\right)\right\}+\mathbf{M}_{t_{k}} \mathbf{F}_{t_{k}}^{-1}\left(\mathbf{y}_{t_{k+1}}-\mathbf{Z}_{t_{k}} \mathrm{E}\left\{\breve{\mathbf{x}}_{\breve{\mathbf{x}}_{t_{k} \mid t_{k}}}\left(t_{k+1} \mid t_{k}\right)\right\}\right) \\
& =\left(\mathbf{I}-\mathbf{M}_{t_{k}} \mathbf{F}_{t_{k}}^{-1} \mathbf{Z}_{t_{k}}\right) \mathrm{E}\left\{\breve{\mathbf{x}}_{\breve{\mathbf{x}}_{t_{k} \mid t_{k}}}\left(t_{k+1} \mid t_{k}\right)\right\}+\mathbf{M}_{t_{k}} \mathbf{F}_{t_{k}}^{-1} \mathbf{y}_{t_{k+1}} .
\end{aligned}
$$

Thus,

$$
\begin{aligned}
& \left|\mathrm{E}\left\{\mathbf{x}_{\breve{\mathbf{x}}_{t_{k} \mid t_{k}}}\left(t_{k+1} \mid t_{k+1}\right)\right\}-\mathrm{E}\left\{\breve{\mathbf{x}}_{\breve{\mathbf{x}}_{t_{k} \mid t_{k}}}\left(t_{k+1} \mid t_{k+1}\right)\right\}\right| \\
& \leq\left|\left(\mathbf{Z}_{t_{k}}^{-1}-\mathbf{M}_{t_{k}} \mathbf{F}_{t_{k}}^{-1}\right) \mathbf{y}_{t_{k+1}}\right|+\left|\left(\mathbf{I}-\mathbf{M}_{t_{k}} \mathbf{F}_{t_{k}}^{-1} \mathbf{Z}_{t_{k}}\right) \mathrm{E}\left\{\breve{\mathbf{x}}_{\breve{\mathbf{x}}_{t_{k} \mid t_{k}}}\left(t_{k+1} \mid t_{k}\right)\right\}\right| \\
& +\left|\mathbf{Z}_{t_{k}}^{-1} \mathrm{E}\left\{\boldsymbol{\varepsilon}_{\breve{\mathbf{x}}_{t_{k} \mid t_{k}}}\left(t_{k+1} \mid t_{k+1}\right)\right\}\right| .
\end{aligned}
$$


Consider the first term first.

$$
\begin{aligned}
\mathbf{Z}_{t_{k}}^{-1}-\mathbf{M}_{t_{k}} \mathbf{F}_{t_{k}}^{-1}= & \mathbf{Z}_{t_{k}}^{-1}-\hat{\mathbf{P}}_{\mathbf{x}, t_{k+1} \mid t_{k}} \mathbf{Z}_{t_{k}}^{-1}\left(\mathbf{Z}_{t_{k}} \hat{\mathbf{P}}_{\mathbf{x}, t_{k+1} \mid t_{k}} \mathbf{Z}_{t_{k}}^{-1}+\mathbf{H}_{t_{k}}\right)^{-1} \\
= & \mathbf{Z}_{t_{k}}^{-1}\left[\mathbf{I}-\mathbf{Z}_{t_{k}} \hat{\mathbf{P}}_{\mathbf{x}, t_{k+1} \mid t_{k}} \mathbf{Z}_{t_{k}}^{-1}\left(\mathbf{Z}_{t_{k}} \hat{\mathbf{P}}_{\mathbf{x}, t_{k+1} \mid t_{k}} \mathbf{Z}_{t_{k}}^{-1}+\mathbf{H}_{t_{k}}\right)^{-1}\right] \\
= & \mathbf{Z}_{t_{k}}^{-1}\left[\left(\mathbf{Z}_{t_{k}} \hat{\mathbf{P}}_{\mathbf{x}, t_{k+1} \mid t_{k}} \mathbf{Z}_{t_{k}}^{-1}+\mathbf{H}_{t_{k}}\right)-\mathbf{Z}_{t_{k}} \hat{\mathbf{P}}_{\mathbf{x}, t_{k+1} \mid t_{k}} \mathbf{Z}_{t_{k}}^{-1}\right] \\
& \times\left(\mathbf{Z}_{t_{k}} \hat{\mathbf{P}}_{\mathbf{x}, t_{k+1} \mid t_{k}} \mathbf{Z}_{t_{k}}^{-1}+\mathbf{H}_{t_{k}}\right)^{-1} \\
= & \mathbf{Z}_{t_{k}}^{-1} \mathbf{H}_{t_{k}}\left(\mathbf{Z}_{t_{k}} \hat{\mathbf{P}}_{\mathbf{x}, t_{k+1} \mid t_{k}} \mathbf{Z}_{t_{k}}^{-1}+\mathbf{H}_{t_{k}}\right)^{-1} \\
= & c \mathbf{Z}_{t_{k}}^{-1} \mathbf{H}_{t_{k}}^{*}\left(\mathbf{Z}_{t_{k}} \hat{\mathbf{P}}_{\mathbf{x}, t_{k+1} \mid t_{k}} \mathbf{Z}_{t_{k}}^{-1}+c \mathbf{H}_{t_{k}}^{*}\right)^{-1}
\end{aligned}
$$

Consider $\mathbf{U}_{t_{k}} \equiv \mathbf{Z}_{t_{k}}^{-1} \mathbf{H}_{t_{k}}^{*}\left(\mathbf{Z}_{t_{k}} \hat{\mathbf{P}}_{\mathbf{x}, t_{k+1} \mid t_{k}} \mathbf{Z}_{t_{k}}^{-1}+c \mathbf{H}_{t_{k}}^{*}\right)^{-1} \mathbf{y}_{t_{k+1}}$. Recall $\mathbf{Z}_{t_{k}}$ and $\mathbf{H}_{t_{k}}^{*}$ are fixed. And $\hat{\mathbf{P}}_{\mathbf{x}, t_{k+1} \mid t_{k}}$ is a combination of linear functions of sigma points and the values of $\mathbf{g}\left(\mathbf{x}^{*}\right)$ where $\mathbf{x}^{*}$ s are sigma points. Further, sigma points are the continuous functions of $h$. Thus, the Euclidean norm of $\mathbf{U}_{t_{k}}$ is a continuous function of $h$. Since $h$ is bounded with $0<b \leq h \leq 1$ and $\left|\mathbf{y}_{t_{k+1}}\right|$ is also bounded, so is $\left|\mathbf{U}_{t_{k}}\right|$. Therefore, $c\left|\mathbf{U}_{t_{k}}\right| \leq K b^{p}$. Similarly, we have

$$
\begin{aligned}
(\mathbf{I} & \left.-\mathbf{M}_{t_{k}} \mathbf{F}_{t_{k}}^{-1} \mathbf{Z}_{t_{k}}\right) \\
= & \mathbf{Z}_{t_{k}}^{-1}\left[\mathbf{I}-\mathbf{Z}_{t_{k}} \hat{\mathbf{P}}_{\mathbf{x}, t_{k+1} \mid t_{k}} \mathbf{Z}_{t_{k}}^{-1}\left(\mathbf{Z}_{t_{k}} \hat{\mathbf{P}}_{\mathbf{x}, t_{k+1} \mid t_{k}} \mathbf{Z}_{t_{k}}^{-1}+\mathbf{H}_{t_{k}}\right)^{-1}\right] \mathbf{Z}_{t_{k}} \\
= & \mathbf{Z}_{t_{k}}^{-1}\left[\left(\mathbf{Z}_{t_{k}} \hat{\mathbf{P}}_{\mathbf{x}, t_{k+1} \mid t_{k}} \mathbf{Z}_{t_{k}}^{-1}+\mathbf{H}_{t_{k}}\right)-\mathbf{Z}_{t_{k}} \hat{\mathbf{P}}_{\mathbf{x}, t_{k+1} \mid t_{k}} \mathbf{Z}_{t_{k}}^{-1}\right] \\
& \times\left(\mathbf{Z}_{t_{k}} \hat{\mathbf{P}}_{\mathbf{x}, t_{k+1} \mid t_{k}} \mathbf{Z}_{t_{k}}^{-1}+\mathbf{H}_{t_{k}}\right)^{-1} \mathbf{Z}_{t_{k}} \\
= & c \mathbf{Z}_{t_{k}}^{-1} \mathbf{H}_{t_{k}}^{*}\left(\mathbf{Z}_{t_{k}} \hat{\mathbf{P}}_{\mathbf{x}, t_{k+1} \mid t_{k}} \mathbf{Z}_{t_{k}}^{-1}+c \mathbf{H}_{t_{k}}^{*}\right)^{-1} \mathbf{Z}_{t_{k}} \\
\Rightarrow & \left|\left(\mathbf{I}-\mathbf{M}_{t_{k}} \mathbf{F}_{t_{k}}^{-1} \mathbf{Z}_{t_{k}}\right) \mathrm{E}\left\{\breve{\mathbf{x}}_{\breve{\mathbf{x}}_{t_{k} \mid t_{k}}}\left(t_{k+1} \mid t_{k}\right)\right\}\right| \leq K b^{p} .
\end{aligned}
$$

Since $\left|\varepsilon_{t_{k}}\right| \leq K b^{p}$ and $\mathbf{Z}_{t_{k}}$ is fixed, $\left|\mathbf{Z}_{t_{k}}^{-1} \mathrm{E}\left\{\boldsymbol{\varepsilon}_{\breve{\mathbf{x}}_{t_{k} \mid t_{k}}}\left(t_{k+1} \mid t_{k+1}\right)\right\}\right| \leq K b^{p}$. Thus, we have

$$
\left|\mathrm{E}\left\{\mathbf{x}_{\breve{\mathbf{x}}_{t_{k} \mid t_{k}}}\left(t_{k+1} \mid t_{k+1}\right)\right\}-\mathrm{E}\left\{\breve{\mathbf{x}}_{\breve{\mathbf{x}}_{t_{k} \mid t_{k}}}\left(t_{k+1} \mid t_{k+1}\right)\right\}\right| \leq K b^{p} \leq K h^{p}
$$

for $0<b \leq h \leq 1$. Therefore, Condition 2 is satisfied. 
- Let's consider Condition 2 of Theorem 5. We have

$$
\begin{aligned}
&\left|\mathrm{E}\left\{\mathbf{x}_{\breve{x}_{t_{k} \mid t_{k}}}\left(t_{k+1} \mid t_{k+1}\right)\right\}-\mathrm{E}\left\{\breve{\mathbf{x}}_{t_{k} \mid t_{k}}\right\}\right| \\
&=\mid \mathrm{E}\left\{\mathbf{x}_{\breve{\mathbf{x}}_{t_{k} \mid t_{k}}}\left(t_{k+1} \mid t_{k+1}\right)\right\}-\mathrm{E}\left\{\breve{\mathbf{x}}_{\breve{\mathbf{x}}_{t_{k} \mid t_{k}}}\left(t_{k+1} \mid t_{k+1}\right)\right\} \\
&\left.+\mathrm{E}\left\{\breve{\mathbf{x}}_{\breve{x}_{t_{k}} \mid t_{k}}\left(t_{k+1} \mid t_{k+1}\right)\right\}-\mathrm{E}\left\{\breve{\mathbf{x}}_{t_{k} \mid t_{k}}\right\}\right\} \mid \\
& \leq\left|\mathrm{E}\left\{\mathbf{x}_{\breve{\mathbf{x}}_{t_{k} \mid t_{k}}}\left(t_{k+1} \mid t_{k+1}\right)\right\}-\mathrm{E}\left\{\breve{\mathbf{x}}_{\breve{x}_{t_{k} \mid t_{k}}}\left(t_{k+1} \mid t_{k+1}\right)\right\}\right| \\
&\left.+\mid \mathrm{E}\left\{\breve{\mathbf{x}}_{\breve{x}_{t_{k} \mid t_{k}}}\left(t_{k+1} \mid t_{k+1}\right)\right\}-\mathrm{E}\left\{\breve{\mathbf{x}}_{t_{k} \mid t_{k}}\right\}\right\} \mid \\
& \leq\left.K\left(1+\left|\mathrm{E}\left\{\breve{\mathbf{x}}_{t_{k} \mid t_{k}}\right\}\right|\right) h^{p}+\mid \mathrm{E}\left\{\breve{\mathbf{x}}_{\breve{\mathbf{x}}_{t_{k} \mid t_{k}}}\left(t_{k+1} \mid t_{k+1}\right)\right\}-\mathrm{E}\left\{\breve{\mathbf{x}}_{t_{k} \mid t_{k}}\right\}\right\} \mid,
\end{aligned}
$$

where the last inequality is from Condition 1. By linear projection, we have

$$
\begin{aligned}
& \left.\mid \mathrm{E}\left\{\breve{\mathbf{x}}_{\breve{\mathbf{x}}_{t_{k} \mid t_{k}}}\left(t_{k+1} \mid t_{k+1}\right)\right\}-\mathrm{E}\left\{\breve{\mathbf{x}}_{t_{k} \mid t_{k}}\right\}\right\} \mid \\
& =\mid \mathrm{E}\left\{\breve{\mathbf{x}}_{\breve{\mathbf{x}}_{t_{k} \mid t_{k}}}\left(t_{k+1} \mid t_{k}\right)\right\}+\mathbf{M}_{t_{k}} \mathbf{F}_{t_{k}}^{-1}\left(\mathbf{y}_{t_{k+1}}-\mathbf{Z}_{t_{k}} \mathrm{E}\left\{\breve{\mathbf{x}}_{\breve{\mathbf{x}}_{t_{k} \mid t_{k}}}\left(t_{k+1} \mid t_{k}\right)\right\}\right) \\
& \left.\quad-\mathrm{E}\left\{\breve{\mathbf{x}}_{t_{k} \mid t_{k}}\right\}\right\} \mid .
\end{aligned}
$$

Let's consider $\mathbf{M}_{t_{k}} \mathbf{F}_{t_{k}}^{-1}\left(\mathbf{y}_{t_{k+1}}-\mathbf{Z}_{t_{k}} \mathrm{E}\left\{\breve{\mathbf{x}}_{\breve{\mathbf{x}}_{t_{k} \mid t_{k}}}\left(t_{k+1} \mid t_{k}\right)\right\}\right)$ first. Note that

$$
\mathbf{M}_{t_{k}} \mathbf{F}_{t_{k}}^{-1}=\hat{\mathbf{P}}_{\mathbf{x}, t_{k+1} \mid t_{k}} \mathbf{Z}_{t_{k}}^{-1}\left(\mathbf{Z}_{t_{k}} \hat{\mathbf{P}}_{\mathbf{x}, t_{k+1} \mid t_{k}} \mathbf{Z}_{t_{k}}^{-1}+\mathbf{H}_{t_{k}}\right)^{-1}
$$

Assuming a fixed initial value, $\hat{\mathbf{P}}_{\mathbf{x}, t_{k+1} \mid t_{k}}=h \mathbf{P}_{t_{k+1} \mid t_{k}}^{*}$ for some covariance matrix $\mathbf{P}_{t_{k+1} \mid t_{k}}^{*}$. Also $\mathbf{P}_{t_{k+1} \mid t_{k}}^{*}$ can be expressed as the sum and product of the values of bounded functions of the form $g(\cdot)$ from the UT. Thus, all entries of $\mathbf{P}_{t_{k+1} \mid t_{k}}^{*}$ are also bounded for all $k$ and so are $\left(\mathbf{Z}_{t_{k}} \hat{\mathbf{P}}_{\mathbf{x}, t_{k+1} \mid t_{k}} \mathbf{Z}_{t_{k}}^{-1}+\mathbf{H}_{t_{k}}\right)^{-1}$ 's. Since $|\mathbf{g}|$ is bounded and $\left|\mathbf{y}_{t_{k+1}}\right|$ are bounded, $\left|\left(\mathbf{y}_{t_{k+1}}-\mathrm{E}\left\{\breve{x}_{\breve{x}_{t_{k}} \mid t_{k}}\left(t_{k+1} \mid t_{k}\right)\right\}\right)\right|$ is bounded. Thus, we get

$$
\begin{aligned}
& \left|\mathrm{E}\left\{\breve{\mathbf{x}}_{\breve{\mathbf{x}}_{t_{k} \mid t_{k}}}\left(t_{k+1} \mid t_{k+1}\right)\right\}-\mathrm{E}\left\{\breve{\mathbf{x}}_{t_{k} \mid t_{k}}\right\}\right| \\
& \leq\left|\mathrm{E}\left\{\breve{\mathbf{x}}_{\breve{\mathbf{x}}_{t_{k}} \mid t_{k}}\left(t_{k+1} \mid t_{k}\right)\right\}-\mathrm{E}\left\{\breve{\mathbf{x}}_{t_{k} \mid t_{k}}\right\}\right|+K h \\
& \leq\left|\mathrm{E}\left\{\breve{\mathbf{x}}_{\breve{\mathbf{x}}_{t_{k}} \mid t_{k}}\left(t_{k} \mid t_{k}\right)\right\}+h \mathrm{E}\left\{\breve{g}\left(\breve{\mathbf{x}}_{\breve{\mathbf{x}}_{t_{k}} \mid t_{k}}\left(t_{k} \mid t_{k}\right)\right)\right\}-\mathrm{E}\left\{\breve{\mathbf{x}}_{t_{k} \mid t_{k}}\right\}\right|+K h \\
& =\left|\mathrm{E}\left\{\breve{\mathbf{g}}\left(\breve{\mathbf{x}}_{t_{k} \mid t_{k}}\right)\right\}\right| h+K h \\
& \leq K h,
\end{aligned}
$$


where $\mathrm{E}\left\{\breve{\mathbf{g}}\left(\breve{\mathbf{x}}_{t_{k} \mid t_{k}}\right)\right\}$ is the mean calculated from the UT. Therefore,

$$
\begin{aligned}
\left.\mid \mathrm{E}\left\{\breve{\mathbf{x}}_{\breve{\mathbf{x}}_{t_{k} \mid t_{k}}}\left(t_{k+1} \mid t_{k+1}\right)\right\}-\mathrm{E}\left\{\breve{\mathbf{x}}_{t \mid t}\right\}\right\} \mid & \leq K\left(1+\left|\mathrm{E}\left\{\breve{\mathbf{x}}_{t_{k} \mid t_{k}}\right\}\right|\right) h^{p}+K h \\
& \leq K\left(1+\left|\mathrm{E}\left\{\breve{\mathbf{x}}_{t_{k} \mid t_{k}}\right\}\right|\right) h .
\end{aligned}
$$

Hence, Condition 2 is satisfied.

- Let's consider Condition 3 of Theorem 5. For $b \leq h \leq 1$, we have

$$
\begin{aligned}
& \left|\mathrm{E}\left\{\mathbf{x}_{\mathbf{x}_{t_{k} \mid t_{k}}}\left(t_{k+1} \mid t_{k+1}\right)\right\}-\mathrm{E}\left\{\mathbf{x}_{\breve{\mathbf{x}}_{t_{k} \mid t_{k}}}\left(t_{k+1} \mid t_{k+1}\right)\right\}\right| \\
& =\left|\mathbf{Z}_{t_{k}}^{-1}\left[\mathbf{y}_{t_{k+1}}-\mathrm{E}\left\{\varepsilon_{\mathbf{x}_{t_{k} \mid t_{k}}}\left(t_{k+1} \mid t_{k+1}\right)\right\}-\mathbf{y}_{t_{k+1}}+\mathrm{E}\left\{\varepsilon_{\breve{\mathbf{x}}_{t_{k} \mid t_{k}}}\left(t_{k+1} \mid t_{k+1}\right)\right\}\right]\right| \\
& \leq K h^{p} .
\end{aligned}
$$

Therefore, Condition 3 is satisfied.

- ii) We do not observe $\mathbf{y}_{t_{k+1}}$.

- Let's consider Condition 1 of Theorem 5. Since we do not have an observation at time $t_{k+1}$, the information up to time $t_{k+1}$ is the same as the information up to time $t_{k}$. Thus,

$$
\begin{aligned}
& \left|\mathrm{E}\left\{\mathbf{x}_{\breve{\mathbf{x}}_{t_{k} \mid t_{k}}}\left(t_{k+1} \mid t_{k+1}\right)\right\}-\mathrm{E}\left\{\breve{\mathbf{x}}_{\breve{\mathbf{x}}_{t_{k} \mid t_{k}}}\left(t_{k+1} \mid t_{k+1}\right)\right\}\right| \\
& =\left|\mathrm{E}\left\{\mathbf{x}_{\breve{\mathbf{x}}_{t_{k} \mid t_{k}}}\left(t_{k+1} \mid t_{k}\right)\right\}-\mathrm{E}\left\{\breve{\mathbf{x}}_{\breve{\mathbf{x}}_{t_{k} \mid t_{k}}}\left(t_{k+1} \mid t_{k}\right)\right\}\right| .
\end{aligned}
$$

This is the norm of an one-step prediction error. So by the same argument as in Section 6, we can obtain

$$
\left|\mathrm{E}\left\{\mathbf{x}_{\breve{\mathbf{x}}_{t_{k} \mid t_{k}}}\left(t_{k+1} \mid t_{k}\right)\right\}-\mathrm{E}\left\{\breve{\mathbf{x}}_{\breve{\mathbf{x}}_{t_{k} \mid t_{k}}}\left(t_{k+1} \mid t_{k}\right)\right\}\right| \leq K h^{3}
$$

Thus, we can obtain $p$ up to 3 . Thus, Condition 1 is satisfied.

- Let's consider Condition 2. By the similar argument as in the proof of Condition 2 in case i),

$$
\begin{aligned}
& \left|\mathrm{E}\left\{\mathbf{x}_{\breve{\mathbf{x}}_{t_{k} \mid t_{k}}}\left(t_{k+1} \mid t_{k+1}\right)\right\}-\mathrm{E}\left\{\breve{\mathbf{x}}_{t_{k} \mid t_{k}}\right\}\right| \\
& =\left|\mathrm{E}\left\{\mathbf{x}_{\breve{\mathbf{x}}_{t_{k} \mid t_{k}}}\left(t_{k+1} \mid t_{k}\right)\right\}-\mathrm{E}\left\{\breve{\mathbf{x}}_{t_{k} \mid t_{k}}\right\}\right| \\
& \leq K\left(1+\left|\mathrm{E}\left\{\breve{\mathbf{x}}_{t_{k} \mid t_{k}}\right\}\right|\right) h .
\end{aligned}
$$

Thus, Condition 2 is satisfied. 
- Let's consider Condition 3. We have

$$
\begin{aligned}
&\left|\mathrm{E}\left\{\mathbf{x}_{\mathbf{x}_{t_{k} \mid t_{k}}}\left(t_{k+1} \mid t_{k+1}\right)\right\}-\mathrm{E}\left\{\mathbf{x}_{\breve{\mathbf{x}}_{t_{k} \mid t_{k}}}\left(t_{k+1} \mid t_{k+1}\right)\right\}\right| \\
&=\left|\mathrm{E}\left\{\mathbf{x}_{\mathbf{x}_{t_{k} \mid t_{k}}}\left(t_{k+1} \mid t_{k}\right)\right\}-\mathrm{E}\left\{\mathbf{x}_{\breve{\mathbf{x}}_{t_{k}} \mid t_{k}}\left(t_{k+1} \mid t_{k}\right)\right\}\right| \\
& \leq\left|\mathrm{E}\left\{\mathbf{x}_{\mathbf{x}_{t_{k} \mid t_{k}}}\left(t_{k+1} \mid t_{k+1}\right)\right\}-\mathrm{E}\left\{\mathbf{x}_{\breve{\mathbf{x}}_{t_{k}} \mid t_{k}}\left(t_{k} \mid t_{k}\right)\right\}\right| \\
&+h\left|\mathrm{E}\left\{g\left(\mathbf{x}_{\mathbf{x}_{t_{k} \mid t_{k}}}\left(t_{k} \mid t_{k}\right)\right)\right\}-\mathrm{E}\left\{g\left(\mathbf{x}_{\breve{x}_{t_{k} \mid t_{k}}}\left(t_{k} \mid t_{k}\right)\right)\right\}\right| \\
&=\left|\mathrm{E}\left\{\mathbf{x}_{t_{k} \mid t_{k}}\right\}-\mathrm{E}\left\{\breve{\mathbf{x}}_{t_{k} \mid t_{k}}\right\}\right|+h\left|\mathrm{E}\left\{g\left(\mathbf{x}_{t_{k} \mid t_{k}}\right)-g\left(\breve{\mathbf{x}}_{t_{k} \mid t_{k}}\right)\right\}\right| .
\end{aligned}
$$

Let $\mathbf{x}_{t_{k} \mid t_{k}}=\left(x_{1, t_{k} \mid t_{k}}, \ldots, x_{c, t_{k} \mid t_{k}}\right)^{T}$. Upon Taylor expansion,

$$
\begin{aligned}
g\left(\mathbf{x}_{t_{k} \mid t_{k}}\right)= & g\left(\mathrm{E}\left\{\mathbf{x}_{t_{k} \mid t_{k}}\right\}\right)+\left(\left(x_{1, t_{k} \mid t_{k}}-\mathrm{E}\left\{x_{1, t_{k} \mid t_{k}}\right\}\right) \frac{\partial}{\partial x_{1}}+\cdots\right. \\
& \left.+\left(x_{c, t_{k} \mid t_{k}}-\mathrm{E}\left\{x_{m, t_{k} \mid t_{k}}\right\}\right) \frac{\partial}{\partial x_{c}}\right)\left.g\right|_{\mathrm{E}\left\{\mathbf{x}_{t_{k} \mid t_{k}}\right\}} \\
& +\frac{1}{2 !}\left(\left(x_{1, t_{k} \mid t_{k}}-\mathrm{E}\left\{x_{1, t_{k} \mid t_{k}}\right\}\right) \frac{\partial}{\partial x_{1}}+\cdots\right. \\
& \left.+\left(x_{m, t_{k} \mid t_{k}}-\mathrm{E}\left\{x_{m, t_{k} \mid t_{k}}\right\}\right) \frac{\partial}{\partial x_{c}}\right)\left.^{2} g\right|_{\boldsymbol{\alpha}} \\
g\left(\breve{\mathbf{x}}_{t_{k} \mid t_{k}}\right)= & g\left(\mathrm{E}\left\{\mathbf{x}_{t_{k} \mid t_{k}}\right\}\right)+\left(\left(\breve{x}_{1, t_{k} \mid t_{k}}-\mathrm{E}\left\{x_{1, t_{k} \mid t_{k}}\right\}\right) \frac{\partial}{\partial x_{1}}+\cdots\right. \\
& \left.+\left(\breve{x}_{c, t_{k} \mid t_{k}}-\mathrm{E}\left\{x_{c, t_{k} \mid t_{k}}\right\}\right) \frac{\partial}{\partial x_{c}}\right)\left.g\right|_{\mathrm{E}\left\{\mathbf{x}_{t_{k} \mid t_{k}}\right\}} \\
& +\frac{1}{2 !}\left(\left(\breve{x}_{1, t_{k} \mid t_{k}}-\mathrm{E}\left\{x_{\left.\left.1, t_{k} \mid t_{k}\right\}\right)} \frac{\partial}{\partial x_{1}}+\cdots\right.\right.\right. \\
& \left.+\left(\breve{x}_{c, t_{k} \mid t_{k}}-\mathrm{E}\left\{x_{c, t_{k} \mid t_{k}}\right\}\right) \frac{\partial}{\partial x_{c}}\right)\left.^{2} g\right|_{\boldsymbol{\beta}}
\end{aligned}
$$

where $\alpha_{i}$ is between $x_{i, t_{k} \mid t_{k}}$ and $\mathrm{E}\left\{x_{i, t_{k} \mid t_{k}}\right\}$ and $\beta_{j}$ is between $\breve{x}_{j, t_{k} \mid t_{k}}$ and $\mathrm{E}\left\{x_{j, t_{k} \mid t_{k}}\right\}$ for $1 \leq i, j \leq c$. Consider

$$
\begin{aligned}
& \left|\mathrm{E}\left[\left(\left(x_{1, t_{k} \mid t_{k}}-\mathrm{E}\left\{x_{1, t_{k} \mid t_{k}}\right\}\right) \frac{\partial}{\partial x_{1}}+\cdots+\left(x_{c, t_{k} \mid t_{k}}-\mathrm{E}\left\{x_{c, t_{k} \mid t_{k}}\right\}\right) \frac{\partial}{\partial x_{c}}\right)^{2} g||_{\alpha}\right]\right| \\
& \leq \sum_{i, j}\left|\mathrm{E}\left[\left.\left(x_{i, t_{k} \mid t_{k}}-\mathrm{E}\left\{x_{i, t_{k} \mid t_{k}}\right\}\right)\left(x_{j, t_{k} \mid t_{k}}-\mathrm{E}\left\{x_{j, t_{k} \mid t_{k}}\right\}\right) \frac{\partial^{2}}{\partial x_{i} \partial x_{j}} g\right|_{\alpha}\right]\right|
\end{aligned}
$$

Since $\mathbf{y}_{t_{k}}$ 's are bounded for all $k, \mathbf{x}_{t_{k}}$ 's are also bounded because of $\mathbf{y}_{t_{k}}=\mathbf{Z}_{t_{k}} \mathbf{x}_{t_{k}}+$ $\varepsilon_{t_{k}}$ where $\left|\varepsilon_{t_{k}}\right| \leq K b^{p}$. Thus, $\left(x_{i, t_{k} \mid t_{k}}-\mathrm{E}\left\{x_{i, t_{k} \mid t_{k}}\right\}\right)$ is bounded for $1 \leq i \leq c$ and 
all $k$. In addition, $\left|\partial^{2} g / \partial x_{i} \partial x_{j}\right| \leq K h^{p-1}$. Therefore, we obtain

$$
\begin{aligned}
& \mid \mathrm{E}\left[\left(\left(x_{1, t_{k} \mid t_{k}}-\mathrm{E}\left\{x_{1, t_{k} \mid t_{k}}\right\}\right) \frac{\partial}{\partial x_{1}}+\cdots\right.\right. \\
& \left.\left.\quad+\left(x_{c, t_{k} \mid t_{k}}-\mathrm{E}\left\{x_{c, t_{k} \mid t_{k}}\right\}\right) \frac{\partial}{\partial x_{c}}\right)\left.^{2} g\right|_{\boldsymbol{\alpha}}\right] \mid \leq K h^{p-1}, \\
& \mid \mathrm{E}\left[\left(\left(\breve{x}_{1, t_{k} \mid t_{k}}-\mathrm{E}\left\{x_{1, t_{k} \mid t_{k}}\right\}\right) \frac{\partial}{\partial x_{1}}+\cdots\right.\right. \\
& \left.\left.\quad+\left(\breve{x}_{c, t_{k} \mid t_{k}}-\mathrm{E}\left\{x_{c, t_{k} \mid t_{k}}\right\}\right) \frac{\partial}{\partial x_{c}}\right)\left.^{2} g\right|_{\boldsymbol{\beta}}\right] \mid \leq K h^{p-1} .
\end{aligned}
$$

Then,

$$
\begin{aligned}
& \left|\mathrm{E}\left\{g\left(\mathbf{x}_{t_{k} \mid t_{k}}\right)\right\}-\mathrm{E}\left\{g\left(\breve{\mathbf{x}}_{t_{k} \mid t_{k}}\right)\right\}\right| \\
& =\mid\left(\left(\mathrm{E}\left\{x_{1, t_{k} \mid t_{k}}\right\}-\mathrm{E}\left\{\breve{x}_{1, t_{k} \mid t_{k}}\right\}\right) \frac{\partial}{\partial x_{1}}+\cdots\right. \\
& \left.\quad+\left(\mathrm{E}\left\{x_{c, t_{k} \mid t_{k}}\right\}-\mathrm{E}\left\{\breve{x}_{c, t_{k} \mid t_{k}}\right\}\right) \frac{\partial}{\partial x_{c}}\right)\left.\mathbf{g}\right|_{\mathrm{E}\left\{\mathbf{x}_{t_{k} \mid t_{k}}\right\}}+K h^{p-1} \\
& \leq K\left|\mathrm{E}\left\{\mathbf{x}_{t_{k} \mid t_{k}}\right\}-\mathrm{E}\left\{\breve{\mathbf{x}}_{t_{k} \mid t_{k}}\right\}\right|+K h^{p-1}
\end{aligned}
$$

where the inequality holds by the Cauchy-Schwarz inequality and the boundedness of the first derivative of $g$. Thus, Condition 3 is satisfied.

Next, we show the conditions of Theorem 6 to complete the UKF-CLS example. Since $\mathbf{y}_{t_{k}}=\mathbf{Z}_{t_{k}} \mathbf{x}_{t_{k}}+\varepsilon_{t_{k}}$, we have

$$
\begin{aligned}
\mathrm{E}\left\{\mathbf{y}_{\mathbf{x}_{t_{k} \mid t_{k}}}\left(t_{k+1} \mid t_{k}\right)\right\} & =\mathbf{Z}_{t_{k}} \mathrm{E}\left\{\mathbf{x}_{\mathbf{x}_{t_{k} \mid t_{k}}}\left(t_{k+1} \mid t_{k}\right)\right\}, \\
\mathrm{E}\left\{\mathbf{y}_{\breve{\mathbf{x}}_{t_{k} \mid t_{k}}}\left(t_{k+1} \mid t_{k}\right)\right\} & =\mathbf{Z}_{t_{k}} \mathrm{E}\left\{\mathbf{x}_{\breve{\mathbf{x}}_{t_{k} \mid t_{k}}}\left(t_{k+1} \mid t_{k}\right)\right\}, \\
\mathrm{E}\left\{\breve{\mathbf{y}}_{\breve{\mathbf{x}}_{t_{k} \mid t_{k}}}\left(t_{k+1} \mid t_{k}\right)\right\} & =\mathbf{Z}_{t_{k}} \mathrm{E}\left\{\breve{\mathbf{x}}_{\breve{\mathbf{x}}_{t_{k} \mid t_{k}}}\left(t_{k+1} \mid t_{k}\right)\right\} .
\end{aligned}
$$

Thus, Condition 1 and 3 of Theorem 6 are satisfied by the Cauchy-Schwarz inequality. Condition 2 and 4 of Theorem 6 are shown in the proofs of Condition 1 and 3 of Theorem 5 , respectively. Therefore, if $\varepsilon_{t_{k}}$ is bounded and its radius is small, we can have a UKF-CLS estimator close to the exact CLS estimator.

\section{REFERENCES}

Ahn, K. W., and Chan, K. S. (2011), "On the convergence rate of the unscented transformation," Submitted, . 
Boyce, W. E., and DiPrima, R. C. (2004), Elementary Differential Equations and Boundary Value Problems, 8 edn, : Wiley.

Chan, K. S., and Kosoy, M. (2010), "Analysis of multi-strain Bartonella pathogens in natural host population - Do they behave as species or minor genetic variants?," Epidemics, $2,165-172$.

Diekmann, O., and Heesterbeek, J. A. P. (2000), Mathematical Epidemiology of Infectious Diseases: Model Building, Analysis and Interpretation, : John Wiley \& Sons Ltd.

Doucet, A., deFreitas, J. F. G., and Gordon, N. J. (2001), Sequential Monte Carlo Methods in Practice, New York: Springer-Verlag.

Durbin, J., and Koopman, S. J. (2000), "Time series analysis of non-Gaussian observations based on state-space models from both classical and Bayesian perspectives (with discussion)," Journal of the Royal Statistical Society Series B, 62, 3-56.

Durbin, J., and Koopman, S. J. (2001), Time Series Analysis by State Space Methods, : Oxford University Press.

Julier, S. J., and Uhlmann, J. K. (1997), "A new extension of the Kalman filter to nonlinear systems," Proc. of AeroSense: The 11th International Symposium on Aerospace/Defense Sensing, Simulation and Controls, Orlando, Florida Vol. Multi Sensor Fusion, Tracking and Resource Management II, .

Julier, S. J., and Uhlmann, J. K. (2004), "Unscented filtering and nonlinear estimation," IEEE Review, 92,, 401-422.

Kalman, R. E. (1960), "A new approach to linear filtering and prediction problems," Journal of Basic Engineering, Transactions ASMA, Series D, 82, 35-45.

Kitagawa, G. (1996), "Monte Carlo filter and smoother for non-Gaussian nonlinear state space models," Journal of Computational and Graphical Statistics, 5, 1-25. 
Klimko, L. A., and Nelson, P. I. (1978), "On conditional least squares estimation for stochastic processes," Annals of Statistics, 6, 629-642.

Kosoy, M. Y., Mandel, E. L., Green, D. C., Marston, E. L., Jones, D. C., and Childs, J. E. (2004), "Prospective studies of Bartonella of rodents. Part I. Demographic and temporal patterns in population dynamics," Vector-Borne and Zoonotic Disease, 4, 285-295.

Liang, H., and Wu, H. (2008), "Parameter estimation for differential equation models using a framework of measurement error in regression models," Journal of American Statistical Association, 103, 1570-1583.

Liu, J. S., and Chen, R. (1998), "Sequential Monte Carlo methods for dynamic systems," Journal of the American Statistical Association, 93, 1032-1044.

Milstein, G. N., and Tretyakov, M. V. (2004), Stochastic Numerics for Mathematical Physics, Berlin Heidelberg, Germany: Springer-Verlag.

Pitt, M., and Shephard, N. (1999), "Filtering via simulation: Auxiliary particle filters," Journal of the American Statistical Association, 94, 590-599.

Ramsay, J. O., Hooker, G., Campbell, D., and Cao, J. (2007), "Parameter estimation for differential equations: A generalized smoothing approach (with discussion)," Journal of the Royal Statistical Society, Series B, 69, 741-796.

Simon, D. (2006), Optimal State Estimation, New Jersey: John Wiley \& Sons, Inc.

van der Vaart, A. W. (1998), Asymptotic Statistics, : Cambridge University Press.

Wu, M., and Smyth, A. W. (2007), "Application of the unscented Kalman filter for real-time nonlinear structural system identification," Structural Control and Health Monitoring, $14,, 971-990$. 